\title{
Focal adhesion kinase plays a dual role in TRAIL resistance and metastatic outgrowth of malignant melanoma
}

\author{
Greta Del Mistro ${ }^{1}$, Shamala Riemann ${ }^{1}$, Sebastian Schindler ${ }^{1,2}$, Stefan Beissert ${ }^{1}$, Roland E. Kontermann ${ }^{3}$, Aurelien Ginolhac ${ }^{4}$,
} Rashi Halder (iD ${ }^{5}$, Luana Presta ${ }^{4}$, Lasse Sinkkonen (D) ${ }^{4}$, Thomas Sauter ${ }^{4}$ and Dagmar Kulms (iD ${ }^{1,2}$

(c) The Author(s) 2022

Despite remarkable advances in therapeutic interventions, malignant melanoma (MM) remains a life-threating disease. Following high initial response rates to targeted kinase-inhibition metastases quickly acquire resistance and present with enhanced tumor progression and invasion, demanding alternative treatment options. We show $2^{\text {nd }}$ generation hexameric TRAIL-receptor-agonist IZI1551 (IZI) to effectively induce apoptosis in MM cells irrespective of the intrinsic BRAF/NRAS mutation status. Conditioning to the $\mathrm{EC}_{50}$ dose of IZI converted the phenotype of IZI-sensitive parental MM cells into a fast proliferating and invasive, IZI-resistant metastasis. Mechanistically, we identified focal adhesion kinase (FAK) to play a dual role in phenotype-switching. In the cytosol, activated FAK triggers survival pathways in a PI3K- and MAPK-dependent manner. In the nucleus, the FERM domain of FAK prevents activation of wtp53, as being expressed in the majority of MM, and consequently intrinsic apoptosis. Caspase-8-mediated cleavage of FAK as well as FAK knockdown, and pharmacological inhibition, respectively, reverted the metastatic phenotype-switch and restored IZI responsiveness. FAK inhibition also re-sensitized MM cells isolated from patient metastasis that had relapsed from targeted kinase inhibition to cell death, irrespective of the intrinsic BRAF/NRAS mutation status. Hence, FAK-inhibition alone or in combination with 2nd generation TRAIL-receptor agonists may be recommended for treatment of initially resistant and relapsed MM, respectively.

Cell Death and Disease (2022)13:54; https://doi.org/10.1038/s41419-022-04502-8

\section{INTRODUCTION}

Targeted therapies as well as immune checkpoint inhibition currently offer a long-term survival to $\sim 30 \%$ of patients with metastatic melanoma (MM). Activating mutations of the serinethreonine kinases NRAS (mutNRAS) or BRAF (mutBRAF) are key drivers of tumor growth through constitutive activation of Mitogen-Activated Protein Kinase (MAPK) pathways involving RAF-MEK-ERK and PI3K-AKT-mTOR, respectively [1-3]. Combination of mutation-specific BRAF inhibitors, and MEK inhibitors show high response rates on mutBRAF MM [4], however, most patients, acquire resistance resulting in tumor relapse $[5,6]$. No targeted therapeutics exist to date to effectively treat mutNRAS MM [7], and response rates to immune checkpoint inhibition remain rather low [8]. Hence, $\sim 70 \%$ of patients still succumb to the disease due to enhanced regrowth of treatment-resistant metastases.

Metastatic outgrowth involves loss of cellular adhesion, and invasive growth through the extracellular matrix (ECM) [9]. Integrins, a family of cell-surface adhesion receptors are composed of $\alpha-$ and $\beta$-subunits that mediate adhesion to the ECM [10, 11]. Upon activation, integrins convey intracellular processes through activation of focal adhesion kinase (FAK/PTK2) and SRC kinase family members to trigger anchorage-independent cell survival, proliferation, and migration [12-14]. Activation of FAK requires a conformational change to uncover its kinase domain. Displacement of the N-terminal FERM domain is facilitated upon interaction with the cytosolic domains of $\beta$-integrins, allowing activation of its kinase function through autocatalytic phosphorylation of Tyr397 [15]. In the cytosol, activated FAK interacts with a variety of cytoskeletal proteins and upstream kinases including SRC, PI3K, and PDK1 to mediate downstream activation of RAFMEK-ERK and PI3K-AKT-(mTOR) signaling pathways [16, 17]. A fraction of FAK, however, translocates into the nucleus where its FERM domain interacts with the N-terminal domain of wildtype p53 (wtp53) and MDM2 to facilitate p53 degradation thereby preventing apoptosis [17-19]. Accordingly, FAK may play an important role in metastatic progression and apoptosis inhibition in wtp53-expressing MM.

Aiming to identify alternative treatment options for primary and relapsed $\mathrm{MM}$ we recently showed activation of the tumor necrosis factor-related apoptosis-inducing ligand (TRAIL)-receptor with a $2^{\text {nd }}$ generation hexameric TRAIL-receptor agonist (IZI1551 $[20,21])$, to be superior in subjecting mutBRAF MM cells to cell death compared to combined mutBRAF/MEK inhibition [22]. In the present study we discovered that acquired IZI1551-resistance of

\footnotetext{
${ }^{1}$ Experimental Dermatology, Department of Dermatology, TU-Dresden, 01307 Dresden, Germany. ${ }^{2}$ National Center for Tumor Diseases Dresden, TU-Dresden, 01307 Dresden, Germany. ${ }^{3}$ Institute of Cell Biology and Immunology and Stuttgart Research Centre Systems Biology, University of Stuttgart, 70569 Stuttgart, Germany. ${ }^{4}$ Department of Life

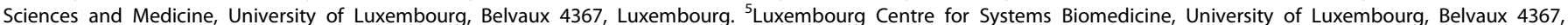
Luxembourg. ${ }^{凶}$ email: dagmar.kulms@uniklinikum-dresden.de

Edited by Hans-Uwe Simon
}

Received: 20 July 2021 Revised: 26 October 2021 Accepted: 7 December 2021

Published online: 12 January 2022 
mutBRAF and mutNRAS MM cells due to long-term exposure to the $E_{50}$ dose of IZI1551 coincides with an enhanced metastatic phenotype, and identified FAK to play a dual role in the observed phenotype-switch.

\section{RESULTS \\ Conditioning to IZI5 renders MM cells resistant to TRAIL- receptor-agonist-induced apoptosis and causes expansion of 3D spheroids}

Based on our hypothesis that tumor cells that do not receive the full lethal, but a suboptimal drug dose may survive treatment, undergo phenotype-switching and cause tumor relapse, we conditioned two mutBRAF (A375, Malme3M) and two mutNRAS (WM1346, WM1366) MM cell lines to the TRAIL-receptor-agonist IZI1551 (IZI), by exposing them to the $\mathrm{EC}_{50}$ dose of $5 \mathrm{ng} / \mathrm{ml}$ (IZI5; [22]) for 6 months. Subsequent, exposure of cells to a lethal IZI dose of $50 \mathrm{ng} / \mathrm{ml}$ (IZI50), exclusively induced apoptosis in parental cells, while protecting the IZI5-conditioned counterparts (Fig. 1A). Accordingly, processing of caspase-8, caspase-3, and PARP only occurred in parental but not in IZI5-conditioned cells (Fig. 1B). Expression of caspase-8 was diminished in IZI5-conditioned cells, implying that only those cells survived conditioning that expressed lower caspase-8 level, and may therefore cause attenuation of IZI50-induced apoptosis [22, 23] (Fig. 1B). Lack of apoptosis induction in response to IZI5-conditioning was confirmed in an in vivo-mimicking 3D setting of GFP-expressing MM spheroids [24] embedded into gel-matrices that allow monitoring of the same spheroid over time. Eight days after continuous IZI50 treatment, tumor mass (green) versus \% PI-positive $\left(\mathrm{PI}^{+}\right)$dead cells (red) was quantified relative to corresponding untreated controls. As expected, IZI50 treatment of parental spheroids resulted in a significantly decreased tumor volume, and an increased amount of dead cells (Fig. 1C). In contrast, IZI5-conditioned spheroids hardly showed PI positivity while, strikingly, three of them (cA375, cWM1346, cWM1366) presented with significantly increased volumes, and outgrowth of cells from the spheroid core (Fig. $1 C)$, implying that long-term exposure to the $\mathrm{EC}_{50}$ dose of IZI1551, confers treatment resistance, and concomitantly drives tumor regrowth and metastatic outgrowth.

\section{Conditioning to IZI5 enhances proliferation of mutBRAF and mutNRAS MM cells}

Increased spheroid volumes implied that IZI5-conditioned cells proliferated faster. Indeed, untreated IZI5-conditioned cells showed significant higher proliferation compared to the individual parental counterparts (Fig. 2A). Accordingly, treatment with IZI5 significantly decreased proliferation of parental cells, being more pronounced upon IZI50 treatment (Fig. 2B, Fig. S1). In contrast, neither exposure to the $\mathrm{EC}_{50}$ nor the lethal IZI dose significantly influenced accelerated proliferation of IZI5conditioned mutBRAF A375 and Malme3M, or mutNRAS WM1366 cells. Only proliferation of mutNRAS WM1346 cells was diminished, but still as high as of untreated parental cells (Fig. 2B, Fig. S1), indicating that the vital status of IZI5-conditioned cells remains largely unaffected upon exposure to the lethal dose.

\section{Conditioning to IZI5 enhances clonogenic outgrowth and migration of mutBRAF and mutNRAS MM cells}

Clonogenic outgrowth was enhanced in three (Malme3M, WM1346, WM1366) out of four untreated IZI5-conditioned compared to parental cells, and remained significantly less affected upon treatment with IZI5 or IZI50, irrespective of the intrinsic mutation status (Fig. 3A).

In order to monitor the effect of IZI5-conditioning on tumor cell migration we performed scratch assay. After $24 \mathrm{~h}$ gap closure was almost complete for GFP-expressing IZI5-conditioned A375, Malme3M and WM1346 cells, whereas only $40-60 \%$ of the gap had been invaded by parental cells (Fig. 3B). Surprisingly, IZI5conditioned WM1366 cells presented with slightly attenuated gap closure compared to parental cells (Fig. 3B).

To this end, we demonstrate that IZI5-conditioned mutBRAF and mutNRAS MM cells (i) remained resistant against the lethal IZI50 dose, (ii) presented with tumor outgrowth in response to IZI50, and iii) showed enhanced proliferation, clonogenic outgrowth, and migration. Notably, not every MM cell pair showed each of the above-mentioned phenotypes, reflecting the heterogeneity of individual tumor specimens that should be considered during the identification of common druggable targets.

\section{Transcriptomic analysis identifies FAK as key regulator of the} metastatic phenotype of IZI5-conditioned MM cells

Aiming to identify common molecular changes in mutBRAF and mutNRAS MM cells that may confer phenotype-switching, we performed RNAseq analysis of parental and IZI5-conditioned IZI50stimulated cells, and used rlog regularized counts for tackling the heteroskedasticity of data for visualization of gene expression ([25]; statistics Table S1). We selected A375 (mutBRAF) and WM1346 (mutNRAS) for further analysis, since they showed all features of phenotype-switching. Gene expression profiles of parental cells revealed pronounced modifications $6 \mathrm{~h}$ after IZI50 stimulation, but remained largely unaffected in IZI5conditioned cells as illustrated by volcano plots (Fig. 4A). Data implied apoptosis resistance as well as enhanced proliferation/ migration to be less dependent on de novo gene synthesis but rather due to changes in pre-existing signal transduction pathways. Venn analysis revealed 1248 intersecting genes to be differentially expressed upon IZI50 treatment in both of the parental, but not IZI5-conditioned cells (Fig. S2). To identify key regulators of consistently affected pathways, we performed GeneWalk analysis, a tool which employs representation learning on gene ontology (GO) information and allows identifying key genes and their relevant functions [26]. GeneWalk-predicted upstream regulators were plotted according to the 'Fraction of Relevant GO Terms per Gene' versus 'Number of GO Annotations per Gene', and the number of direct interaction partners within the regulatory network indicated by the size of the respective dots. Top regulators that are active and relevant are presented in the upper right corner, followed in priority by regulators plotted in the bottom right corner (Fig. 4B). From these two groups of high priority regulators we selected 18 candidates (Table S2) that interacted with 20 or more partners within the regulatory network. Out of these 18 candidates, 10 (MET, PML, PRKDC, RARA, TRAF6, FAK(PTK2), IQGAP1, ITGAV, FN1, RB1) served a function in the regulation of "cell death/survival", "migration/invasion", or "tumorigenesis". However, only six (MET, PML, PRKDC, FAK(PTK2), IQGAP1, ITGAV) showed a meaningful (genes regulated in the context of metastatic phenotype) and coherent (analogous gene regulation) expression pattern in mutBRAF and mutNRAS cells. In particular, focal adhesion kinase FAK(PTK2) caught our attention, because it is capable of conferring cell death resistance and to promote proliferation, migration and invasion of cancer cells $[15,17,27]$. FAK gene expression was coherently upregulated in IZI5-conditioned A375 and WM1346 cells, and pronouncedly downregulated in parental cells in response to IZI50 (Fig. 4C; log2FoldChanges/p-values Table S3). Western-blot analysis did not confirm significant upregulation of FAK protein in IZI5-conditioned cells, but revealed that cleavage of full length $125 \mathrm{kDa}$ FAK into a $\sim 90 \mathrm{kDa}$ fragment exclusively occured in parental cells upon IZI50 treatment (Fig. 4D). FAK cleavage was caspase-dependent, because it could be prevented by pan-caspase inhibition using QVD (Fig. 4E). Specifically, FAK processing appeared to be facilitated by caspase- 8 , because it remained absent in WM115 MM clones \#1 and \#2 in which caspase-8 had been knocked out by CRISPR-Cas9 technology (Fig. 4F). Thus, reduced caspase- 8 expression in IZI5-conditioned cells (Fig. 1B) may contribute to 


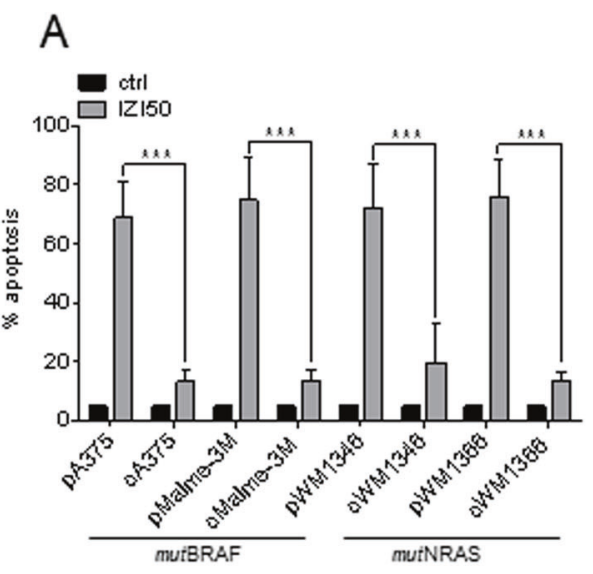

B

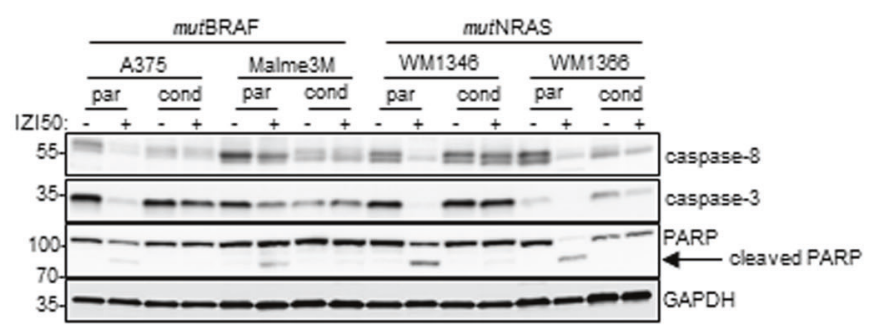

C
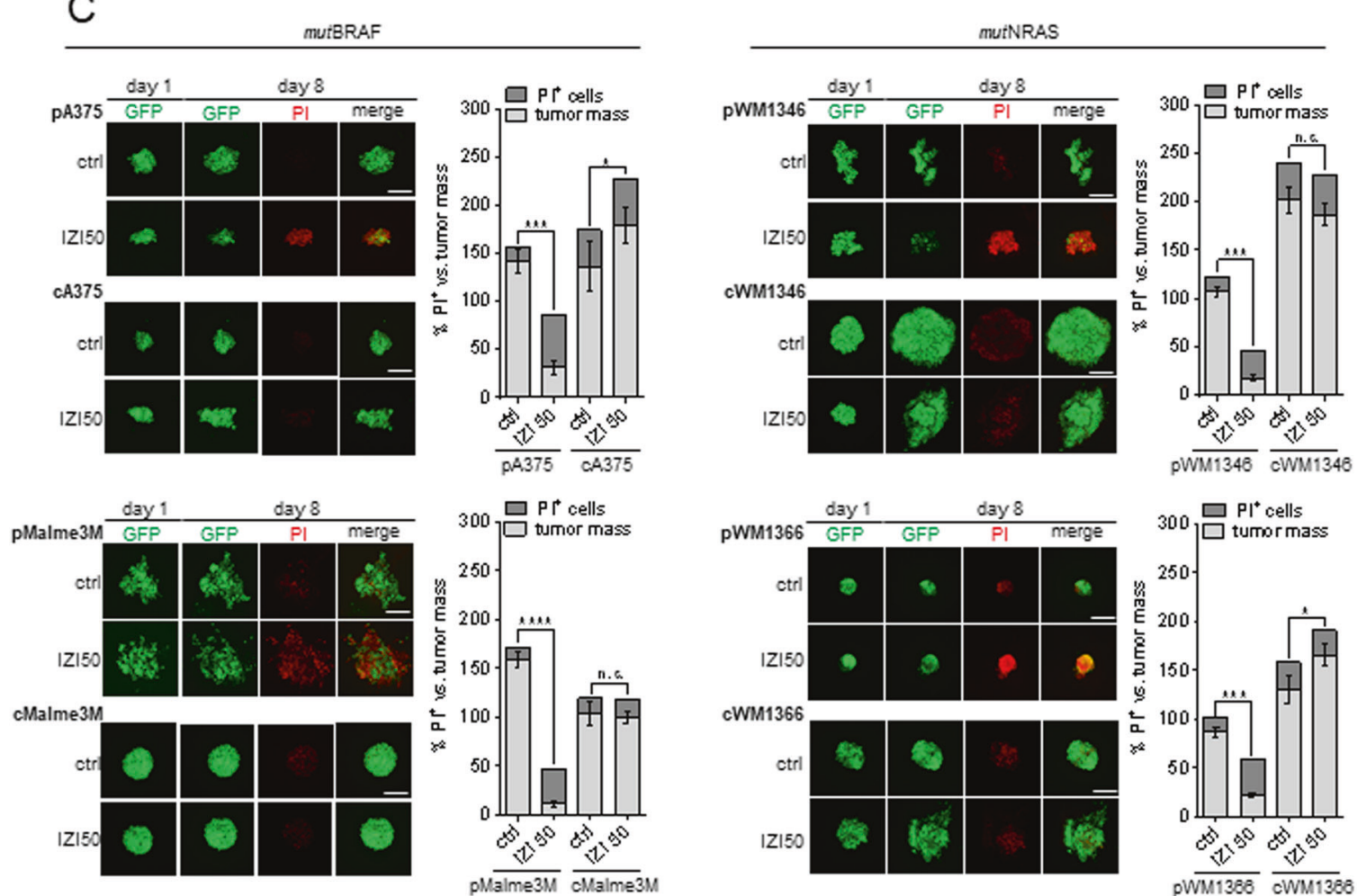

Fig. 1 Conditioning to IZI5 renders MM cells resistant to TRAIL-receptor-agonist-induced apoptosis and causes expansion of 3D spheroids. A Apoptosis induction was monitored $16 \mathrm{~h}$ after stimulation of parental (p) and IZI5-conditioned (c) BRAF-mutated (mutBRAF) A375 and Malme3M as well as NRAS-mutated (mutNRAS) WM1346 and WM1366 MM cells with IZI50 using a Cell Death Detection ELISA (CDDE) $\left(n=3:{ }^{* *} p \leq 0.001\right)$. B Processing of caspase-8, caspase-3, and PARP was assessed by Western-blot analysis with GAPDH as loading control. One representative out of three independently performed experiments is shown. C GFP-expressing green fluorescent spheroids consisting of parental (p) and IZI5-conditioned (c) melanoma cells were individually embedded into 3D dextran-based gel-matrices and stimulated with IZI50 every other day over 8 days (scale bar $=200 \mu \mathrm{m}$ ). At day eight cell death was visualized by addition of $6.7 \mu \mathrm{g} / \mathrm{ml} \mathrm{PI}$. Confocal images of individual spheroids at day one and eight and the quantification of tumor volume (green) versus tumor death (red) at day eight are shown $\left(n=3:{ }^{*} p \leq 0.05 ;{ }^{* * *} p \leq 0.001 ;\right.$ n.s. $=$ not significant).

TRAIL resistance and enhanced migration of MM cells by impeding FAK cleavage.

\section{Integrin-mediated FAK activation causes upregulation of MAPK signaling pathways}

Within the GeneWalk regulatory network, FAK was identified to interact with 62 proteins (Fig. 5A), with 41 serving a function in the regulation of "cell death/survival", "migration/invasion", or "tumorigenesis" (Table S4). Only 25 of these FAK interactors showed a reasonable and coherent expression pattern in both, mutBRAF and
mutNRAS cells. We identified four candidates (PRKDC, MET, IQGAP1, ITGAV) that directly interacted with FAK and additionally presented as top regulators of the signaling network (Fig. 5A, marked in yellow, Fig. 4B). Expression of the catalytic subunit of DNA-dependent protein kinase (PRKDC), the mesenchymalepithelial transition factor (MET), and the Ras GTPase-activatinglike protein (IQGAP1), respectively, was upregulated in IZI5conditioned compared to parental cells, and down-regulation of these regulators of DNA repair, migration, invasion, or cell cycle, was attenuated in IZI5-conditioned cells in response to IZI50 
A
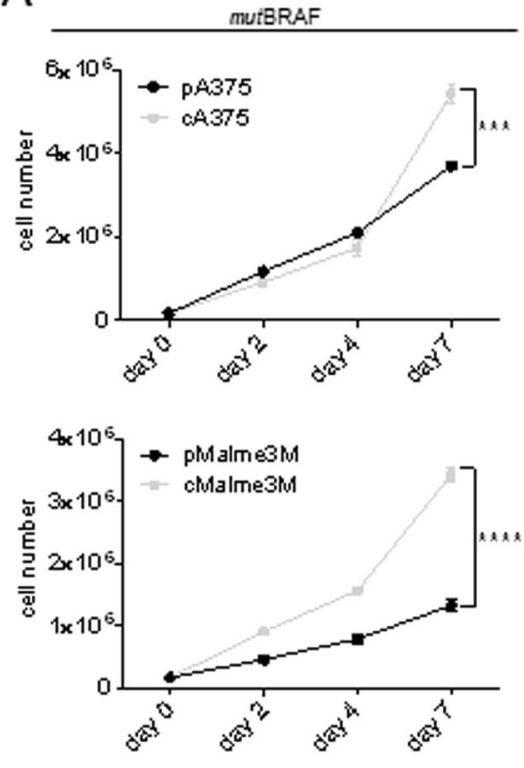

B
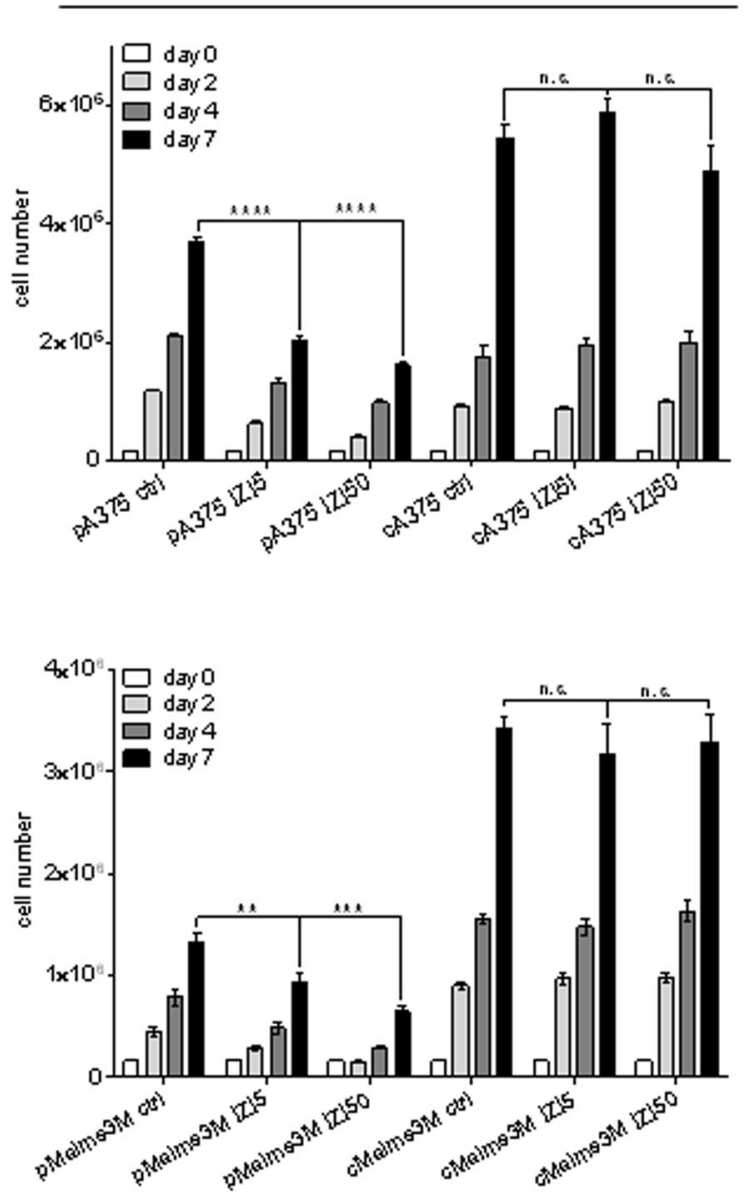

(3)

MUIBRAF
mUINRAS
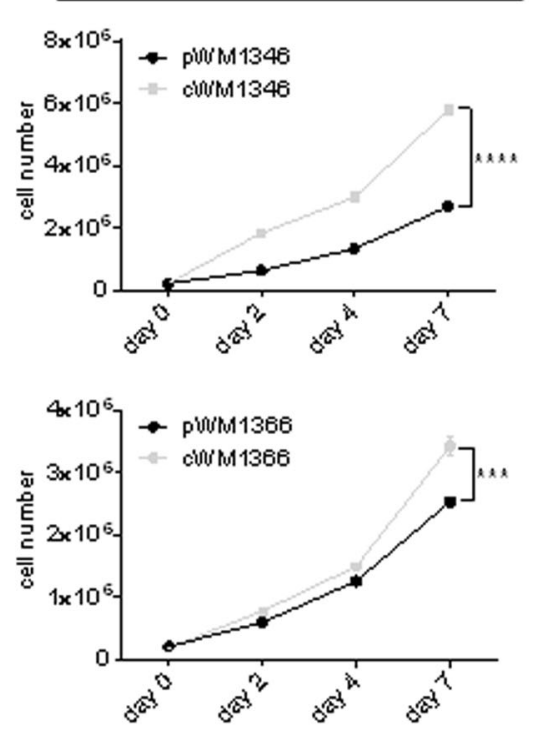
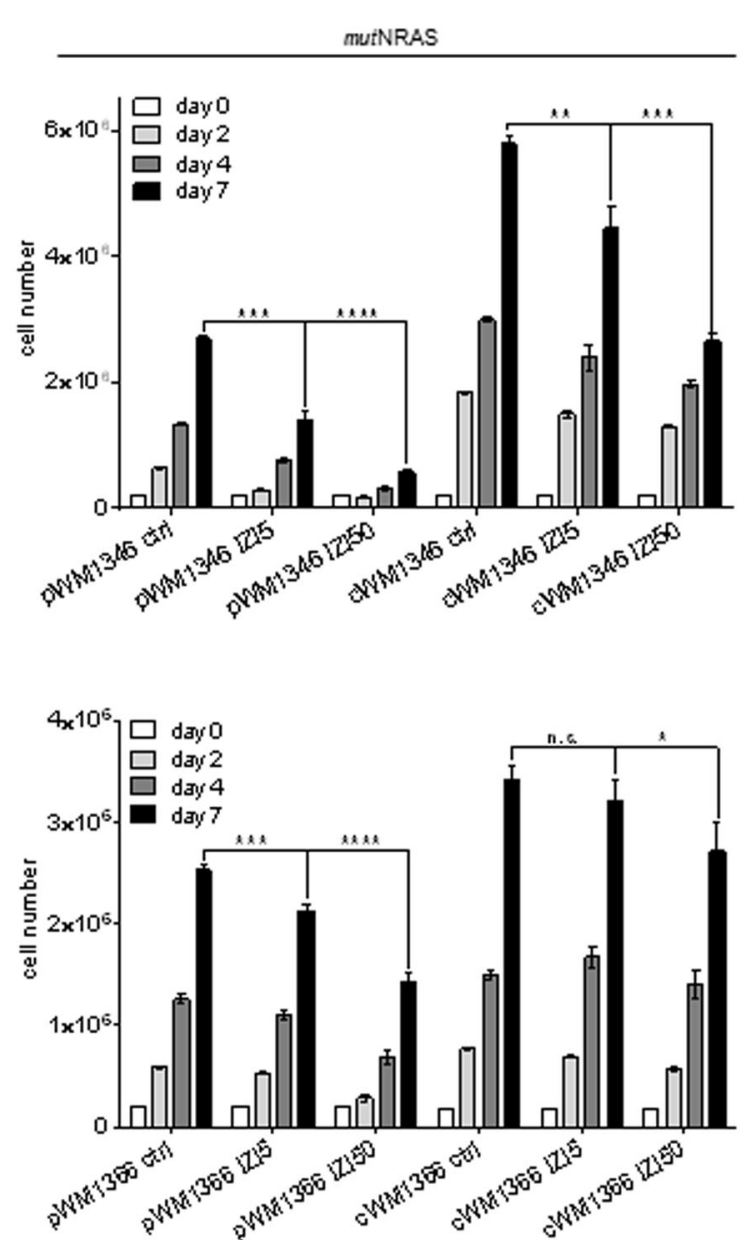

Fig. 2 Conditioning to IZI5 enhances proliferation of mutBRAF and mutNRAS MM cells. A Proliferation of unstimulated parental ( $p$ ) versus IZI5-conditioned $(c, 5 \mathrm{ng} / \mathrm{ml}) \mathrm{MM}$ cells was quantified at the indicated time points $\left({ }^{* * *} p \leq 0.001 ;{ }^{* * *} p \leq 0.0001\right)$. B Parental (p) and IZI5conditioned (c) cells were left untreated or stimulated with IZI5 and IZI50, respectively, every other day. Proliferation of cells was quantified at the indicated time points $\left(n=3:{ }^{*} p \leq 0.05 ;{ }^{* *} p \leq 0.01 ;{ }^{* * *} p \leq 0.001 ;{ }^{* * *} p \leq 0.0001 ;\right.$ n.s. $=$ not significant $)$. 
A
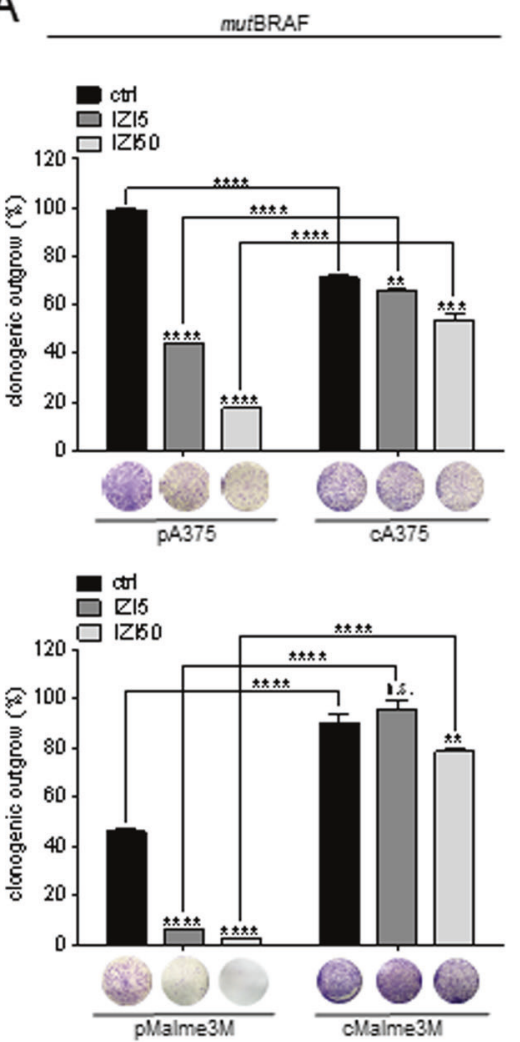

\begin{tabular}{l} 
mutNRAS \\
\hline वri \\
$\square$ Z150
\end{tabular}
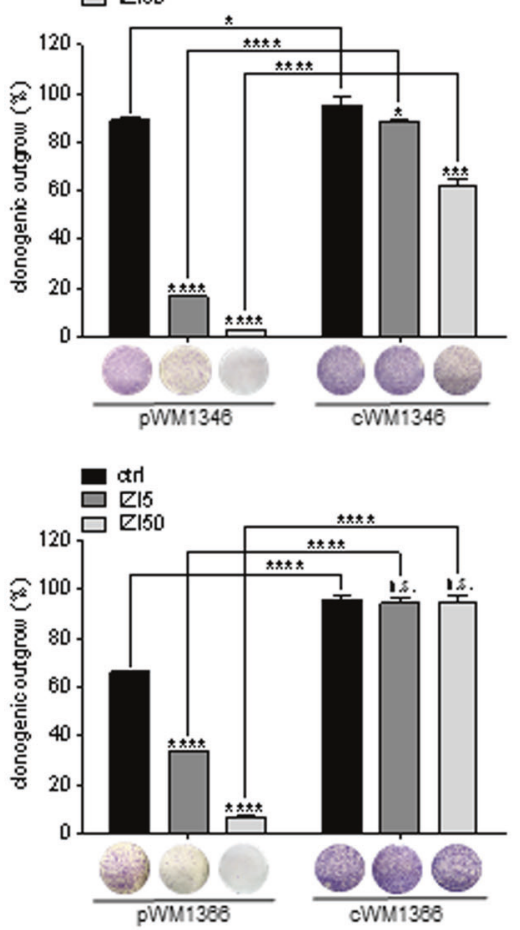

B
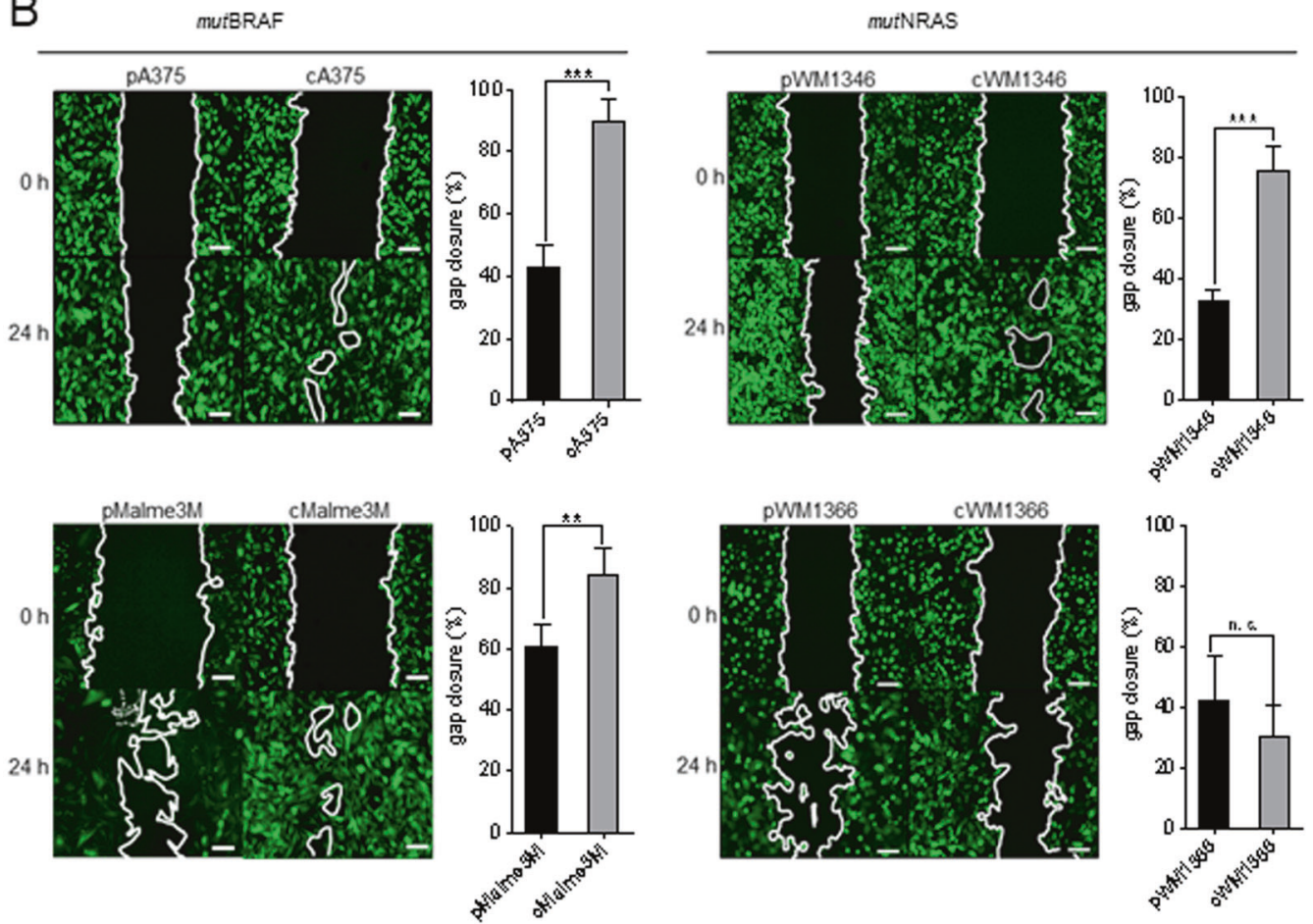

Fig. 3 Conditioning to IZI5 enhances clonogenic outgrowth and migration of mutBRAF and mutNRAS MM cells. A Parental (p) and IZI5conditioned (c) MM cells seeded at $10 \%$ confluency were left untreated or stimulated with IZI5 and IZI50, respectively, every other day. After 8 days, colonies were stained with crystal violet, and absorbance of eluted crystal violet at $595 \mathrm{~nm}$ was quantified $(n=3)\left({ }^{*} p \leq 0.05 ;{ }^{* *} p \leq 0.01\right.$; ${ }^{* * *} p \leq 0.001 ;{ }^{* * * *} p \leq 0.0001 ;$ n.s. = not significant). B Migration of untreated GFP-expressing parental (p) and IZI5-conditioned (c) cells was monitored by scratch assay (scale bar $=100 \mu \mathrm{m})$ and gap closure quantified after $24 \mathrm{~h}\left(n=3:{ }^{* *} p \leq 0.01 ;{ }^{* * *} p \leq 0.001 ;\right.$ n.s. $=$ not significant). 


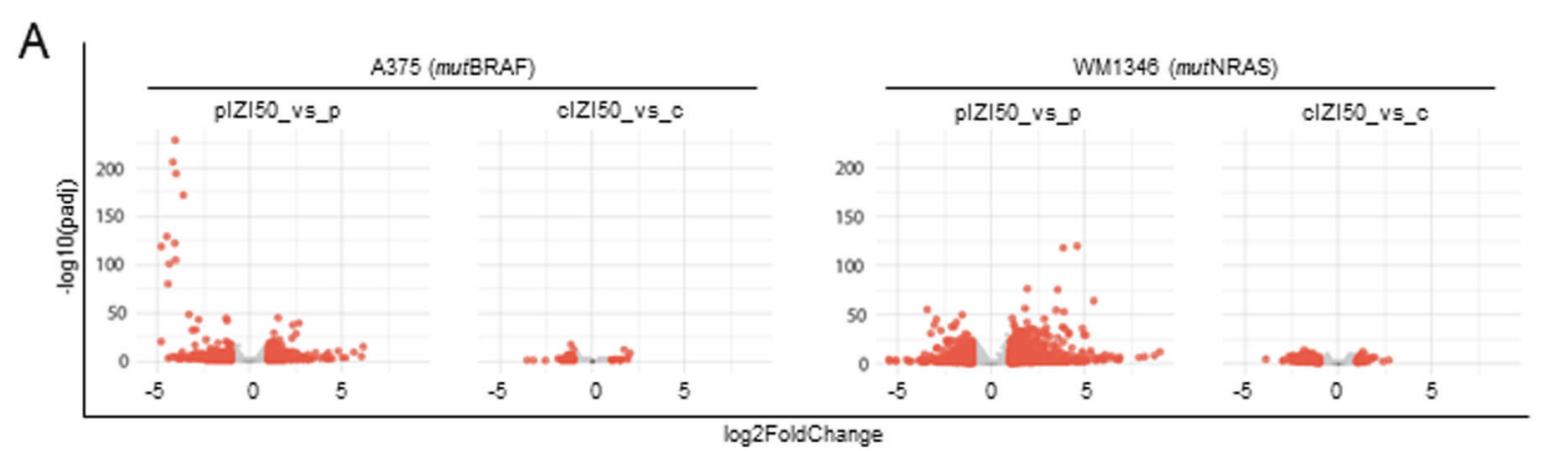

B

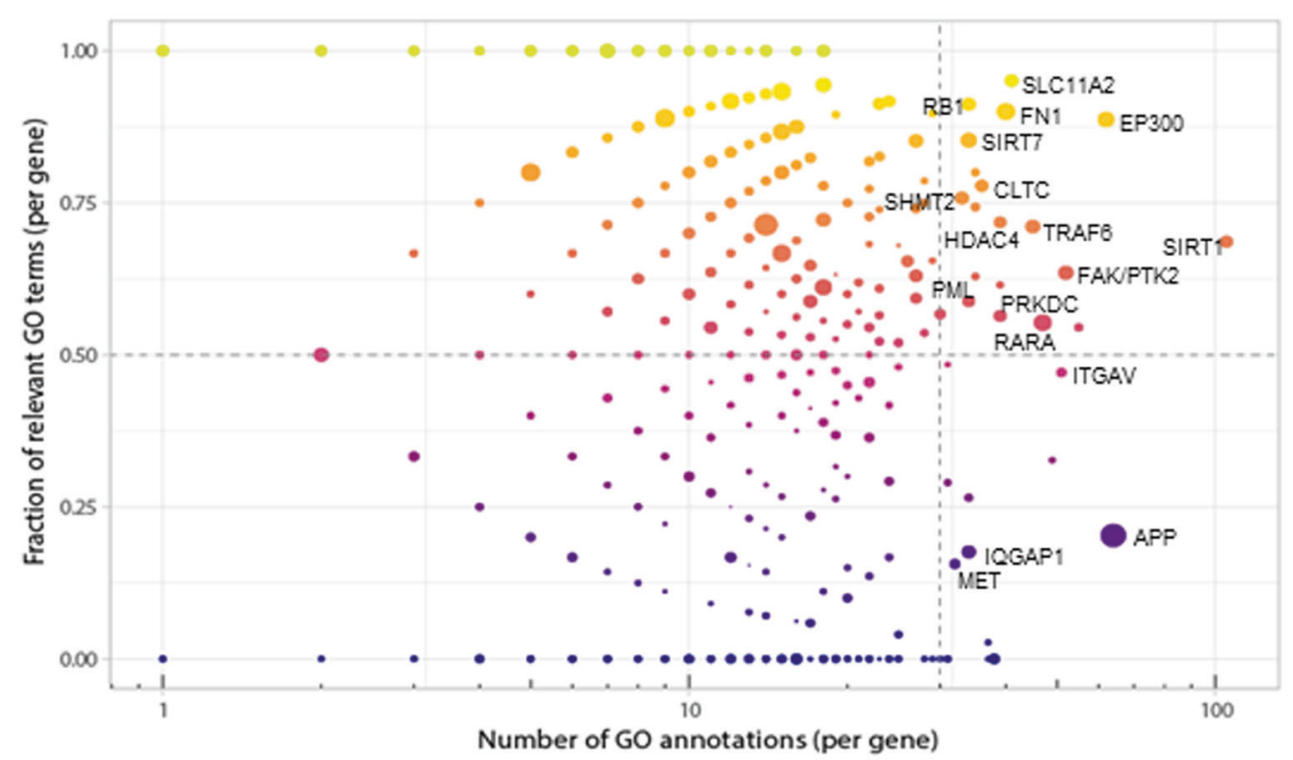

Gene connections - $050 \bullet 100 \bigcirc 150$

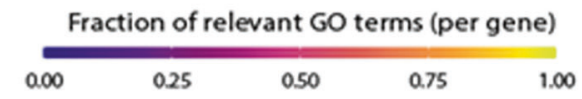
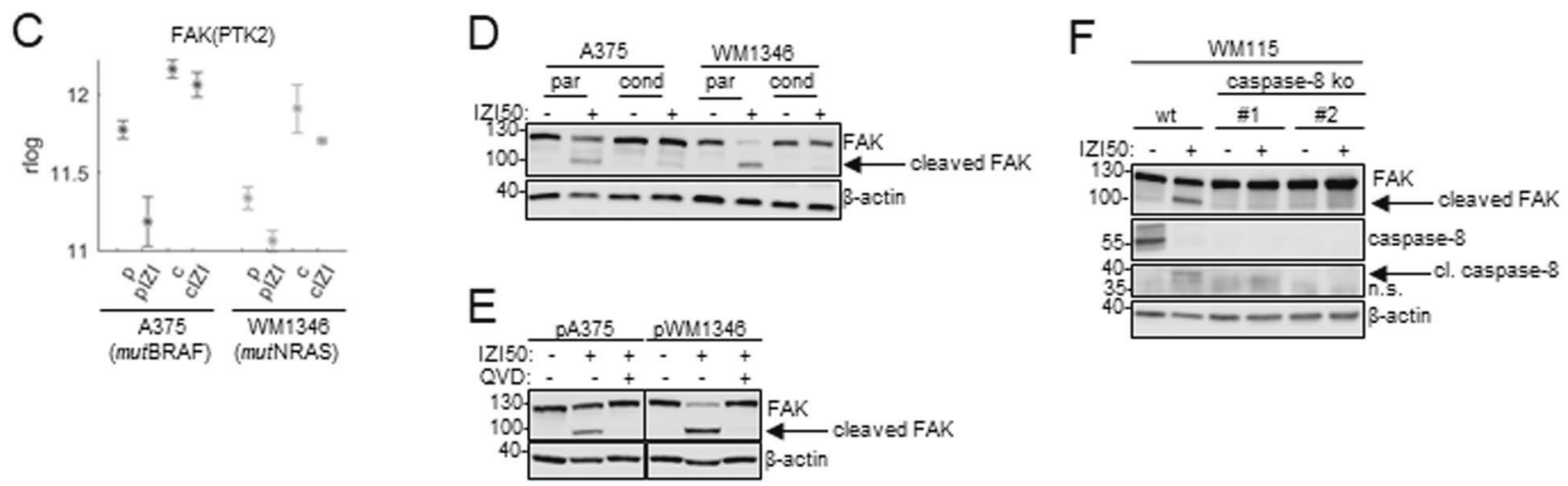

(Fig. 5B; log2FoldChanges/p-values Table S5). Elevated expression of integrin aV subunit (ITGAV) in IZI5-conditioned cells was even enhanced upon IZI50 treatment, making ITGAV the most promising candidate for further analysis (Fig. 5B). Integrin $\mathrm{aV}$ preferably forms heterodimers with the $\beta 3$ subunit to facilitate MM cell motility and cancer progression [10]. Consequently, surface expression of the integrin aV $\beta 3$ heterodimer was significantly elevated in IZI5-conditioned cells (Fig. 5C), and may therefore promote activation of FAK. Accordingly, initial FAK
Tyr397 phosphorylation was markedly enhanced in IZI5conditioned cells and remained unaffected upon IZI50-mediated TRAIL-receptor activation. Where present in parental cells, basal FAK-phosphorylation disappeared upon IZI50 stimulation, most likely due to FAK cleavage (Fig. 5D). In IZI5-conditioned mutBRAF A375 cells FAK activation coincided with PDK1- and PI3K-driven activation of downstream AKT, whereas mutNRAS WM1346 cells responded with PDK1- and PI3K-independent AKT activation as well as enhanced ERK-p38-cJun signaling, any of which is known 
Fig. 4 Transcriptomic analysis identifies FAK as key regulator of the metastatic phenotype of IZI5-conditioned MM cells. A Parental ( $p$ ) and conditioned (c) A375 and WM1346 cells remained untreated or where stimulated with IZI50 for six hrs. Subsequently, RNAseq analysis of three biological replicates was performed and gene expression changes presented by vulcano plots. Genes are represented as dots in -log 10 (adjusted p-value) versus log2 (fold-change). Differentially expressed genes with adjusted $p$-value $\leq 0.1$ and abs (fold-change) $\geq 1$ are marked in red. B Differentially expressed genes with consistent directionality of change in the parental contexts of both cell lines, but not being differentially expressed in the conditioned contexts (1248 genes), were fed into GeneWalk with default settings. Regulators are represented as dots in 'Fraction of Relevant GO Terms per Gene' versus 'Number of GO Annotations per Gene'. C Mean expression (rlog counts) of the FAK (PKT2) gene in parental (p) and IZI5-conditioned (c) A375 and WM1346 cells in response to IZI50 treatment for 6 h. D Parental (p) and IZI5conditioned (c) A375 and WM1346 cells were stimulated with IZI50. After $24 \mathrm{~h}$ processing of FAK was determined by Western-blot analysis. One representative out of three independently performed experiments is shown. E Parental (p) A375 and WM1346 cells were pretreated or not with $5 \mu \mathrm{M}$ QVD for $1 \mathrm{~h}$. Subsequently cells were stimulated with IZI50 and cleavage of FAK monitored $24 \mathrm{~h}$ later by Western-blot analysis. One representative out of three independently performed experiments is shown. $\mathbf{F}$ Wildtype (wt) and caspase-8 knock out (clones \#1 and \#2) WM115 melanoma cells were stimulated with IZI50 and the protein/cleavage status of FAK and caspase- 8 assessed after $24 \mathrm{~h}$ by Western-blot analysis. $\beta$-actin served as loading controls. One representative out of three independently performed experiments is shown.

to promote cell survival, proliferation and migration [16] (Fig. 5D). Hence, FAK may act in concert with or upstream of PI3K, PDK1 and SRC activation, to prevent cell death and concomitantly promote proliferation and migration of MM. Accordingly, re-sensitization of IZI5-conditioned A375 and WM1346 cells was shown to be most pronounced upon inhibition of FAK with defactinib (iFAK), compared to individual inhibition of PI3K (alpelisib), PDK1 (GSK2334470), AKT (afuresertib), and SRC (dasatinib) (Fig. 5E), and showed lowest toxicity towards untransformed cells of the skin (Fig. S3). Consequently, inhibition of FAK may provide a reasonable and tumor selective strategy to sensitize MM to cell death, irrespective of their BRAF/NRAS mutation status.

\section{Nuclear FAK prevents p53 activation in response to IZI50 treatment}

To attribute a dual role in phenotype-switching of IZI5conditioned MM cells to FAK, we investigated the impact of FAK inhibition on both, cell survival and cell death. Defactinib fully abolished phosphorylation of FAK and consequently of PI3K, PDK1, AKT, ERK, p38, cJun, and SRC where applicable, and resulted in processing of caspase-3 and cleavage of PARP (Fig. 6A). In concert with caspase activation, cleavage of FAK occurred, thereby presumably promoting apoptosis in a positive feedback mechanism. Accordingly, re-sensitization of IZI5-conditioned A375 and WM1346 cells to IZI50 by FAK inhibition was completely prevented by the pan-caspase inhibitor QVD, but not by the RIP1-inhibitor Nec1S (Fig. 6B).

Besides its function as a cytosolic tyrosine kinase, nuclear FAK is able to prevent wtp53 accumulation and consequently tumor cell death independent of its kinase function [19]. Hence, a fraction of FAK and caspase-8, but not caspase-3 or caspase-9, localized to the nuclear compartment of both, parental and IZI5-conditioned MM cells (Fig. 6C). In IZI50-stimulated parental cells, activation of caspase- 8 and consequently cleavage of nuclear FAK nicely coincided with an accumulation and activation of p53, being evident by Ser15 phosphorylation. (Fig. 6C). Consequently, upregulation of pro-apoptotic BAK, BAX and PUMA genes (Fig. 6D, log2FoldChanges/p-values Table S6) and intrinsic apoptosis becomes initiated in a caspase-9/caspase-3-dependent manner [28] (Fig. 6C). In IZI5-conditioned cells, integrin-mediated activation of cytosolic FAK triggers proliferation and migration in a MAPK-dependent manner, whereas nuclear FAK prevents wtp53induced apoptosis induction. Upon TRAIL-receptor activation in parental cells, proteolytic activation of caspase-8 causes cleavage of FAK in the cytosol and the nucleus thereby counteracting both functions of FAK, and consequently executing apoptosis.

\section{TRAIL-resistance and tumor outgrowth of IZI5-conditioned MM cells depends on FAK}

To consolidate that phenotype-switching of IZI5-conditioned MM cells specifically depended on FAK, we knocked down FAK in A375 and WM1346 cells by RNA interference (Fig. 7A). FAK depletion fully re-sensitized IZI5-conditioned cells to IZI50-induced apoptosis (Fig. 7B), and significantly reduced proliferation (Fig. 7C). Pharmacological inhibition of FAK with defactinib, significantly reduced migration of IZI5-conditioned cells (Fig. 7D), almost back to the level of parental cells (compare Fig. 3B), and completely abolished outgrowth of cells from as well as increase in the volume of 3D spheroids consisting of any of the four IZI5conditioned MM cells (mutBRAF A375, Malme3M; mutNRAS WM1346, WM1366) in response to IZI50 treatment. Instead, apoptosis was induced as determined by PI incorporation (Fig. 7E, compare Fig. 1B), allowing to conclude that FAK inhibition may universally break TRAIL resistance and prevent invasiveness of MM independent of the intrinsic mutation status.

\section{FAK inhibition sensitizes resistant mutBRAF and mutNRAS melanoma to mutation-specific targeted kinase inhibition} Since cytosolic FAK acts upstream of BRAF/NRAS, and nuclear FAK interferes with the activation of wtp53 as being expressed in the majority of melanomas, we investigated whether FAK inhibition may have an implication in current clinical applications, i.e. resensitizing $M M$ cells that have overcome mutation-specific targeted kinase inhibition to cell death. To mimic the relapse situation we conditioned mutBRAF Malme3M and WM3248 cells to $1 \mu \mathrm{M}$ dabrafenib and mutNRAS SkMel 2 and SkMel147 cells to $1 \mathrm{nM}$ trametinib for 6 months. Subsequently, parental and conditioned cell pairs were treated with the respective mutation-specific kinase inhibitors alone or with the clinically relevant combination of mutBRAF/MEK inhibitors ( $10 \mu \mathrm{M}$ dabrafenib $+10 \mathrm{nM}$ trametinib). In parallel, we applied three different doses of the FAK inhibitor defactinib $(2.5,5,10 \mu \mathrm{M})$ alone or combined with the mutationspecific kinase inhibitor (Fig. 8A). In all cell lines, cell death induction by $10 \mu \mathrm{M}$ defactinib alone was significantly superior to individual or combined mutBRAF/MEK inhibition, as determined by PI uptake (Fig. 8A). Especially in mutBRAF cells about $60-80 \%$ of cell death was achieved compared to only $10-20 \%$ upon dabrafenib/trametinib application. These differences were explicitly lower in mutNRAS cells, nevertheless, combined inhibition of FAK and the individual mutation-specific kinases yielded strongest cell death in all cell lines and caused re-sensitization of conditioned cells irrespective of the intrinsic mutation status (Fig. 8A). Strikingly, FAK inhibition also re-sensitized therapyresistant MM cells, isolated from three mutBRAF (M45, M53, M59) and three mutNRAS (M10, M20, M32) metastasis relapsed from targeted kinase-inhibition. As expected, melanoma samples only poorly responded to treatment with individual or combined mutation-specific kinase inhibitors (Fig. 8B). Exposure to $10 \mu \mathrm{M}$ FAK inhibitor defactinib alone or combined with either dabrafenib or trametinib-depending on the mutation status-yielded $70-80 \%$ cell death in two mutBRAF (M53, M54) and two mutNRAS (M20, M32) melanoma samples, and improved cell death induction in M45 and M10 cells compared to the clinically relevant drug combination (Fig. 8B). Primary melanocytes, 
A

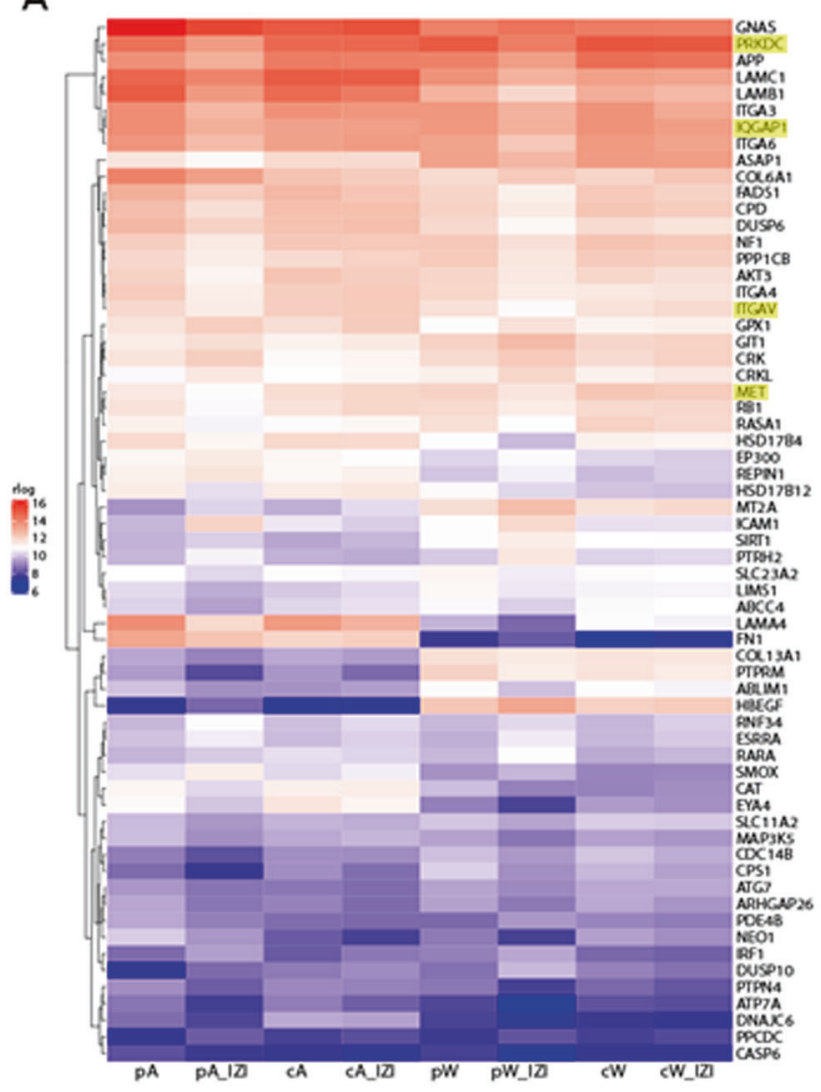

B
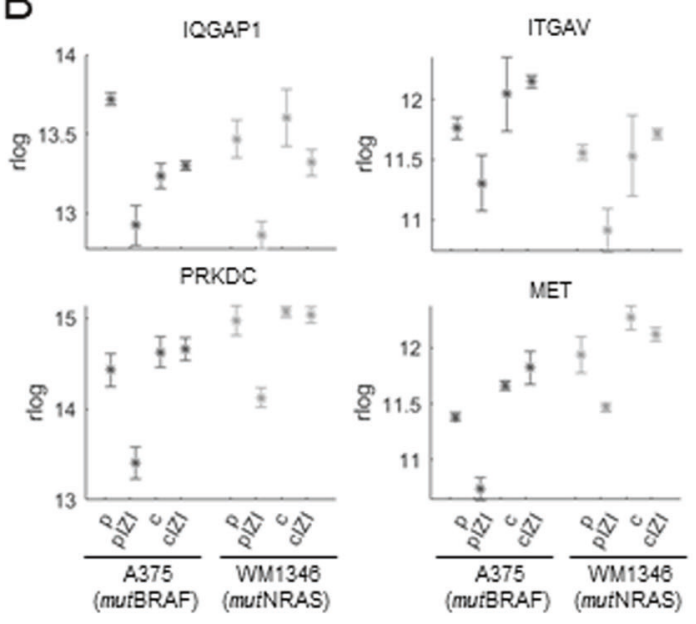

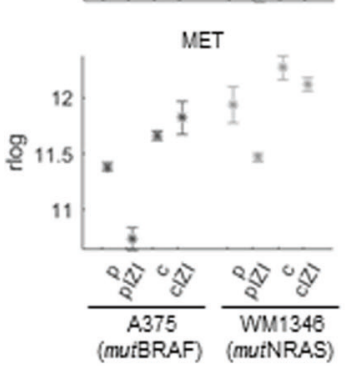

keratinocytes, and fibroblasts remained largely unaffected by the most potent drug combinations, accounting for their tumor selectivity (Fig. S4). Finally, TRAIL-receptor activation using IZI50 was even superior in subjecting all metastatic cell samples to cell death, irrespective of the mutation status, and reached almost
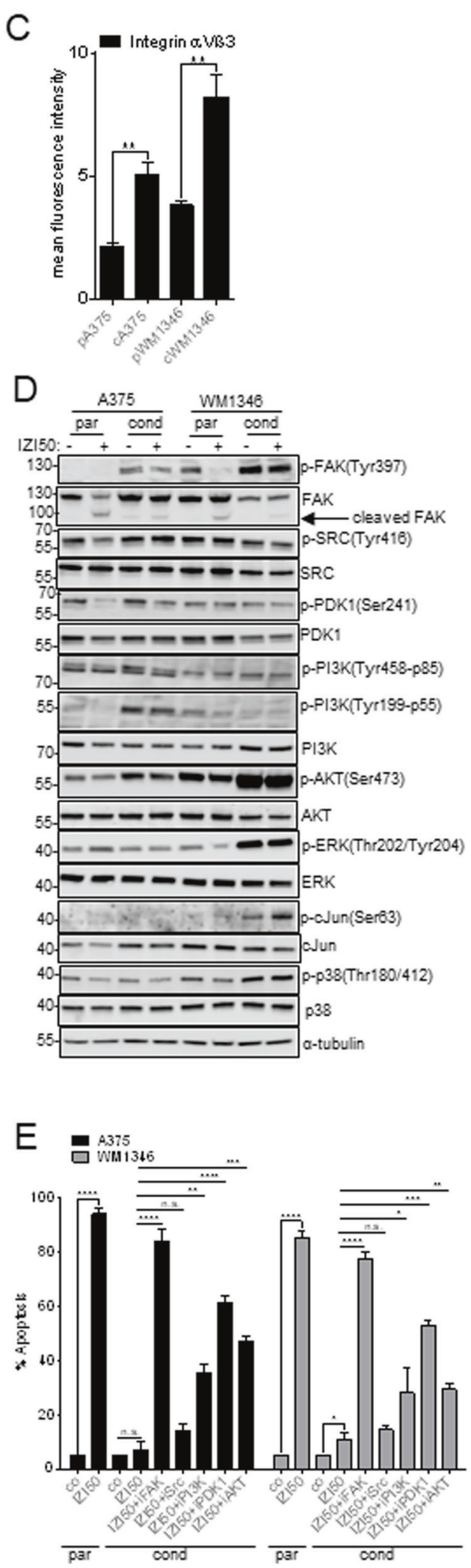

$100 \%$ cell death of targeted kinase inhibitor-resistant mutNRAS MM cells. Taken together, we have identified FAK as a potential target to improve second-line therapy for relapsed MM patients, especially when combined to second generation TRAIL-receptor agonists, like IZI1551. 
Fig. 5 Integrin-mediated FAK activation causes upregulation of MAPK signaling pathways. A Mean expression (rlog counts) of the FAK/ PTK2 interaction partners in the GeneWalk regulatory network in the different contexts of $A 375$ and WM1346 cells. $p=$ parental; $\mathrm{C}=$ conditioned; $\mathrm{IZI}=$ stimulated with $50 \mathrm{ng} / \mathrm{ml} \mathrm{IZI} 1551 ; \mathrm{A}=\mathrm{A} 375 ; \mathrm{W}=\mathrm{WM} 1346$. FAK interaction partners that also represented within the 18 top regulators are marked in yellow. B Mean expression (rlog counts) of PRKDC, IQGAP, ITGAV, MET genes in parental (p) and IZI5-conditioned (c) A375 and WM1346 cells in response to IZI50 treatment for $6 \mathrm{~h}$. C The surface expression of integrin $\alpha \mathrm{V} \beta 3$ on unstimulated parental (p) and IZI5-conditioned (c) A375 and WM1346 cells was determined by flow cytometry ( $\left.n=3:{ }^{* *} p \leq 0.01\right)$. D Parental (p) and IZI5-conditioned (c) A375 and WM1346 cells were stimulated with IZI50. After $24 \mathrm{~h}$ the phosphorylation status of FAK, SRC, PDK1, PI3K, AKT, ERK, CJUN, and p38 was monitored by Western-blot analysis with $\alpha$-tubulin as loading control. One representative out of three independently performed experiments is shown. E Parental (p) A375 and WM1346 cells were stimulated with IZI50. IZI-conditioned (c) cells were treated for $1 \mathrm{~h}$ with the FAK inhibitor (iFAK; $5 \mu \mathrm{M}$ ) defactinib, the SRC inhibitor (iSrc; $10 \mu \mathrm{M}$ ) dasatinib, the PI3K inhibitor (iPI3K; $20 \mu \mathrm{M}$ ) alpelisib, the PDK1 inhibitor (iPDK1; $10 \mu \mathrm{M}$ ) GSK2334470, and the AKT inhibitor (iAKT; $20 \mu \mathrm{M}$ ) afuresertib, respectively, prior to IZI50 stimulation. After $24 \mathrm{~h}$ apoptosis induction was assessed using a CDDE $\left(n=3:{ }^{*} p \leq 0.05 ;{ }^{* *} p \leq 0.01 ;{ }^{* * *} p \leq 0.001 ;{ }^{* * * *} p \leq 0.0001 ;\right.$ n.s. $=$ not significant $)$.

\section{DISCUSSION}

To date, clinical intervention strategies for $\mathrm{MM}$ are exclusively based on the mutation status of key drivers of MM progression, BRAF and NRAS oncogenes. However, other pathophysiological modifications may contribute to therapy resistance and tumor relapse, including tumor cell specific activation of alternative kinases within the signal transduction network [29-32].

Accordingly, high unmet medical need still exists for new treatment options in particular for patients that have acquired resistance to conventional treatment regimens. In this context, $2^{\text {nd }}$ generation TRAIL-receptor agonists with enhanced bioactivity, may overcome the limitations of trimeric TRAIL molecules and receptor-agonistic antibodies that have previously failed in clinical trials [33]. We previously showed hexameric TRAIL-receptoragonist IZI1551 (IZI) $[20,21]$ to be superior in inducing apoptotic cell death in mutBRAF/MEK inhibition-sensitive and -resistant MM cells [22]. However, long-term exposure to the $E_{50}$ dose IZI5, as it may occur within heterogeneous tumor populations, changed the responsiveness to lethal $I Z I 50$, causing acquired resistance and a pronounced metastatic phenotype, involving enhanced proliferation, migration, clonogenic outgrowth, and increased tumor volume. A similar phenotype-switch is also observed upon acquired resistance of $\mathrm{MM}$ against targeted kinase inhibitors in the clinic and hinders second-line treatment regimens [34, 35].

Performing gene ontology analysis with GenWalk we identified FAK as a key player in conferring both apoptosis resistance and an enhanced metastatic phenotype in IZI5-conditioned mutBRAF and mutNRAS MM cells. In concert with integrin-mediated signaling a function in invasion and metastatic characteristics of the epithelial-mesenchymal transition has been attributed to FAK $[13,15]$. Likewise, increased FAK expression has been implemented in survival of other cancer types, including breast cancer [36, 37], bladder cancer [38] and squamous cell carcinoma [39] through activation of MAPK- and PI3K-related signaling pathways [40]. Following this line, we show that MM cells that had acquired resistance to IZI1551, presented with increased integrin aVB3 expression and consequently constitutive FAK activation, followed by downstream activation of MAPK and PI3K-driven survival pathways. In contrast, FAK was found to be largely dephosphorylated and proteolytically cleaved by caspase- 8 in IZI50-stimulated parental cells, thereby preventing the metastatic phenotype and inducing apoptosis. Cleavage of both, caspase-3 and FAK has previously been reported to occur in cisplatinsensitive ovarian cancer cells, and could be prevented upon application of the caspase-3 inhibitor zDEVD [41]. Along this line, sensitization of irradiation-resistant glioma cells with temozolomide antagonized integrin aV $\beta 3$ expression and FAK phosphorylation, causing caspase-3-mediated FAK cleavage and cell death induction [42]. Early studies suggested caspase-mediated cleavage of FAK to contribute to TRAIL-induced cell death [43]. Vice versa, FAK was shown to inhibit death receptor-induced apoptosis through binding to receptor-interacting protein (RIP) [44]. In all these studies it remained, however, unsolved whether FAK cleavage was a cause or a consequence of apoptosis induction.
In this context, de-phosphorylation of FAK was shown to precede its cleavage by caspases in renal epithelial cells [45]. Even though it cannot be fully excluded that caspase- 3 may also cleave FAK in the process of TRAIL-induced cell death, we here show that cytosolic caspase-8-mediated cleavage of FAK is essential for full apoptosis induction in response to TRAIL-receptor activation, because depletion of caspase-8 fully prevented processing of FAK in MM cells that still expressed downstream caspases. Additionally, caspase-8-mediated cleavage of FAK also takes place in the nucleus, in the absence of caspase-3. This is of particular interest, because we could recently show caspase- 8 to be expressed in the nucleus of $\mathrm{MM}$ and other cancer types, where it employs destabilization of $w \operatorname{tp} 53$ and consequently therapy resistance through cleavage of USP28 [46]. Cleavage of FAK resulted in wtp53 activation and induction of intrinsic apoptosis, implying that the FERM domain of cleaved FAK can no longer serve as a scaffold for wtp53/MDM2 interaction. Hence, enhanced FAKmediated wtp53 turnover represents another important mechanism how MM cells, may resist therapeutic intervention [47].

Taken together, we present how FAK may contribute to treatment resistance and a metastatic phenotype in wtp53expressing MM. Cytosolic FAK triggers survival pathways in a PI3K- and MAPK-dependent manner, whereas concomitantly nuclear FAK prevents wtp53 activation. FAK activation can be counteracted through cleavage by caspase- 8 to antagonize both pro-cancerous actions. FAK inhibition reverted the metastatic phenotype-switch evoked by IZI5-conditioning, and restored IZI responsiveness irrespective of the BRAF/NRAS mutation status. FAK inhibition also re-sensitized kinase inhibitor-resistant mutBRAF and mutNRAS MM cell lines as well as patient samples that had relapsed from targeted kinase inhibition. Based on our data FAK-inhibition alone or in combination with $2^{\text {nd }}$ generation TRAILreceptor agonists may be recommended for treatment of primary or therapy resistant relapsed MM metastasis irrespective of the intrinsic mutation status.

\section{MATERIALS AND METHODS}

\section{Cells and reagents}

Human melanoma cell lines (A375, Malme3M, Skmel2, Skmel147, WM115 WM1366, WM1346) were obtained from the American Type Culture Collection (ATCC) and maintained in RPMI 1640 medium (Invitrogen, Karlsruhe, Germany) with $10 \%$ FCS in a humified atmosphere of $5 \% \mathrm{CO}_{2}$ at $37^{\circ} \mathrm{C}$. Primary melanoma cells were freshly isolated from patients metastasis $(\mathrm{M} 10=\mathrm{m}(58) ; \mathrm{M} 20=\mathrm{m}(87) ; \mathrm{M} 32=\mathrm{m}$ (53); $\mathrm{M} 45=\mathrm{m}(41) ;$ $\mathrm{M} 53=\mathrm{m}(73) ; \mathrm{M} 59=\mathrm{m}(48))$ by incubating the chopped tissue samples in HBBS (w/o Ca ${ }^{2+}$ and $\mathrm{Mg}^{2+}$ ) containing $0.05 \%$ collagenase, $0.1 \%$ hyaluronidase, $1.25 \mathrm{U} / \mathrm{ml}$ dispase, $20 \mathrm{mM}$ HEPES, $100 \mathrm{~g} / \mathrm{ml}$ gentamycin, $100 \mathrm{U} / \mathrm{ml}$ penicillin and $100 \mathrm{~g} / \mathrm{ml}$ streptomycin for $60 \mathrm{~min}$ at $37^{\circ} \mathrm{C}$. After centrifugation cell pellets were washed in HBSS/20 mM HEPES and maintained in RPMI $+10 \%$ FCS. The usage of patient material (metastasis) for biochemical analysis was approved by the ethics committee of the TUDresden (EK 335082018) and informed consent was obtained from all patients. Primary cells were purchased from Cell Systems (Troisdorf, Germany) and used at passage 4. Keratinocytes were maintained in Keralife 
A

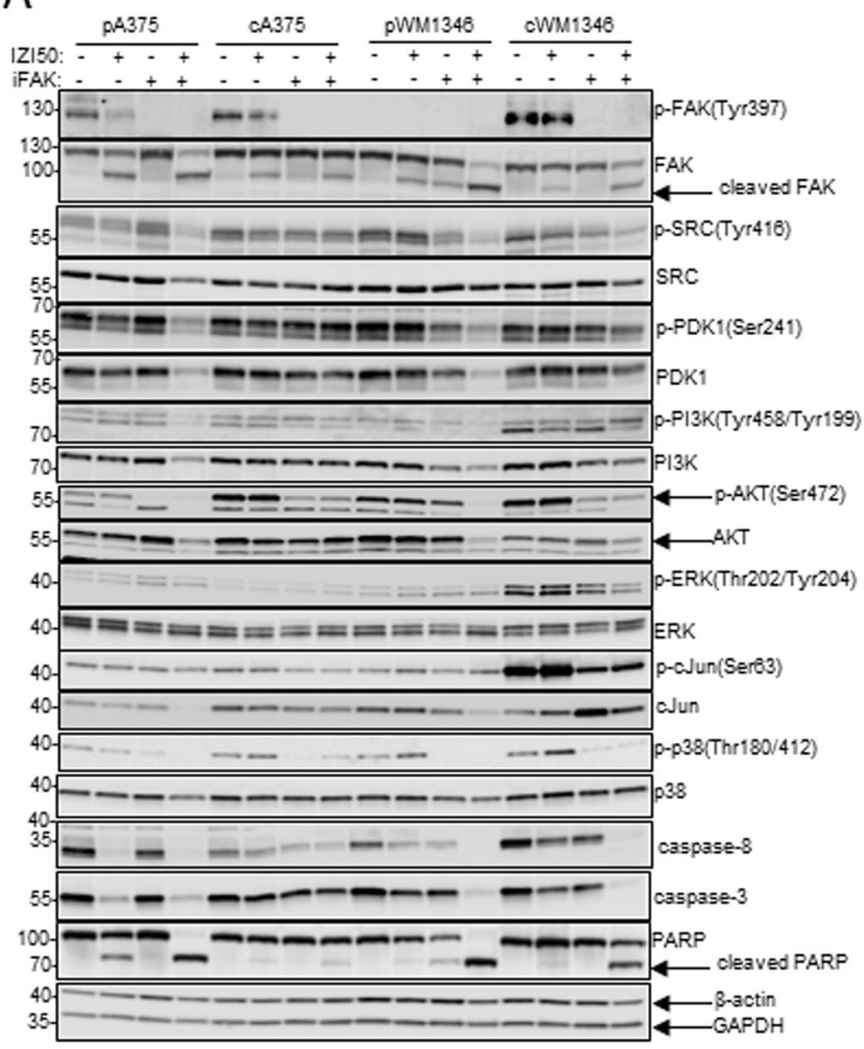

C

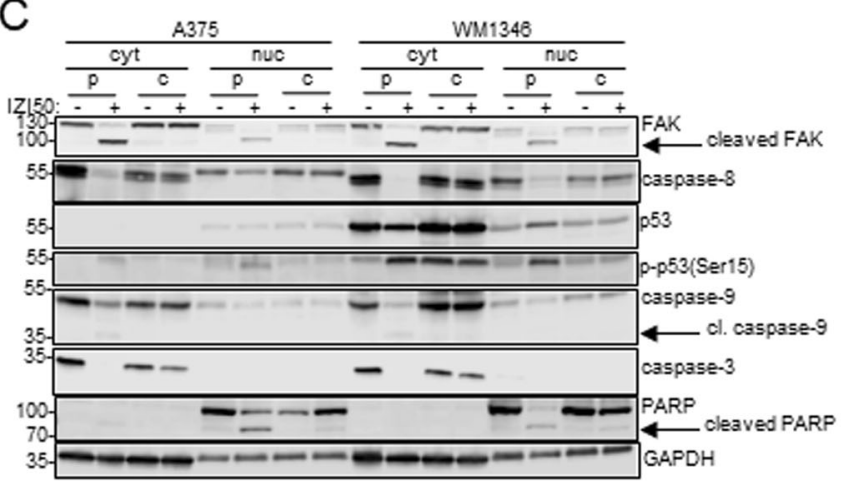

B
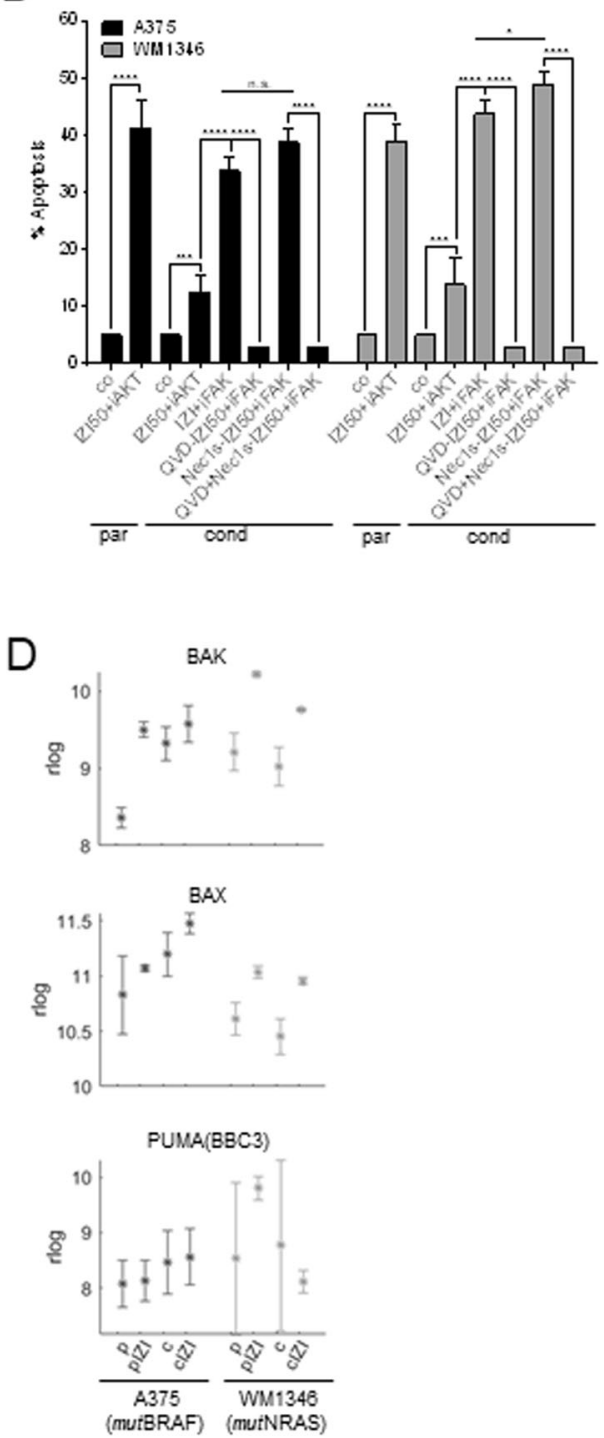

Fig. 6 Nuclear FAK prevents p53 activation in response to IZI50 treatment. A Parental (p) and IZI5-conditioned (c) A375 and WM1346 cells were stimulated with IZI50, $5 \mu \mathrm{M}$ defactinib (iFAK), or both. After $24 \mathrm{~h}$ the phosphorylation status of FAK, SRC, PDK1, PI3K, AKT, ERK, cJUN, and p38, as well as processing of caspase-3 and PARP was monitored by Western-blot analysis with $\beta$-actin and GAPDH as loading controls. One representative out of three independently performed experiments is shown. B Parental (p) A375 and WM1346 cells were stimulated with IZI50. IZI5conditioned (c) cells were treated with either $5 \mu \mathrm{M}$ QVD, $15 \mu \mathrm{M} \mathrm{Nec} 1 \mathrm{~s}$, or both for $1 \mathrm{~h}$ prior to combined FAK inhibitor (iFAK) defactinib and IZI50 stimulation. After $24 \mathrm{~h}$ apoptosis induction was assessed using a CDDE $\left(n=3:{ }^{*} p \leq 0.05 ;{ }^{* * *} p \leq 0.001 ;{ }^{* * *} p \leq 0.0001 ;\right.$ n.s. $=$ not significant). C Parental (p) and IZI5-conditioned (c) A375 and WM1346 cells were stimulated with IZI50. After 24 h the status of FAK, p53, p-p53(Ser15), caspase-8, caspase-3, caspase-9 and PARP, respectively, was determined in cytosolic (cyt) and nuclear (nuc) protein fractions by Western-blot analysis, with GAPDH as loading control. One representative out of three independently performed experiments is shown. D Mean expression (rlog counts) of pro-apoptotic BAK, BAX and PUMA genes in parental (p) and IZI5-conditioned (c) A375 and WM1346 cells in response IZI50 treatment for $6 \mathrm{~h}$.

medium (Cell Systems), fibroblasts in DMEM (Invitrogen), and melanocytes in Melanocyte Growth Medium (M2, Promocell, Heidelberg, Germany). All cell lines were tested every other month to be mycoplasma-negative as judged by the MocyAlert Mycoplasma Detection Kit (LT-07, Lonza, Basel, Switzerland). Cell conditioning was achieved by long-term exposure of melanoma cells to different compounds over a period of 6 months. A375, Malme3M, WM1366, and WM1346 cell lines were conditioned to $5 \mathrm{ng} / \mathrm{ml}$ of the hexavalent TRAIL-receptor-agonist IZI1551 generated at the Institute of Cell Biology and Immunology, University of Stuttgart [20, 21]. WM3248 and Malme3M cell lines were conditioned to $1 \mu \mathrm{M}$ of the mutBRAF inhibitor dabrafenib (\#S2807; Selleckchem, Munich, Germany) and SkMel2 and
SkMel147 cells to $1 \mathrm{nM}$ of the MEK inhibitor trametinib (\#S2673; Selleckchem), respectively.

For stimulation of cells, the pan-caspase inhibitor QVD (Novus Biologicals, Littleton, CO, USA) was added at $5 \mu \mathrm{M}$, the RIP1-inhibitor Nec1S (BioVision, Hannover, Germany) at $15 \mu \mathrm{M}$, the FAK inhibitor defactinib (\#S7654; Selleckchem) at 2.5-10 $\mu \mathrm{M}$, the SRC inhibtitor dasatinib (\#S1021; Sellekchem) at $10 \mu \mathrm{M}$, the PDK1 inhibitor GSK2334470 (\#S7087, Sellckchem) at $20 \mu \mathrm{M}$, the PI3K inhibitor alpelisip (\#HY-15244; MedChemExpress, Moumouth Junction, NJ, USA) at $20 \mu \mathrm{M}$, the AKT inhibitor afuresertib (\#S7521; Selleckchem) at $10 \mu \mathrm{M}$, dabrafenib at $10 \mu \mathrm{M}$, and trametinib at $10 \mathrm{nM}$. 
A

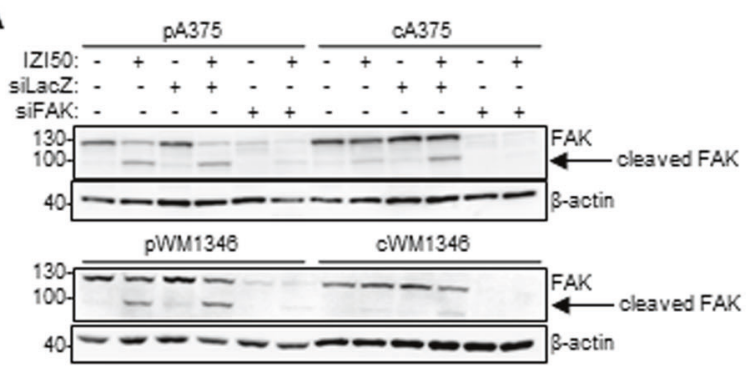

C
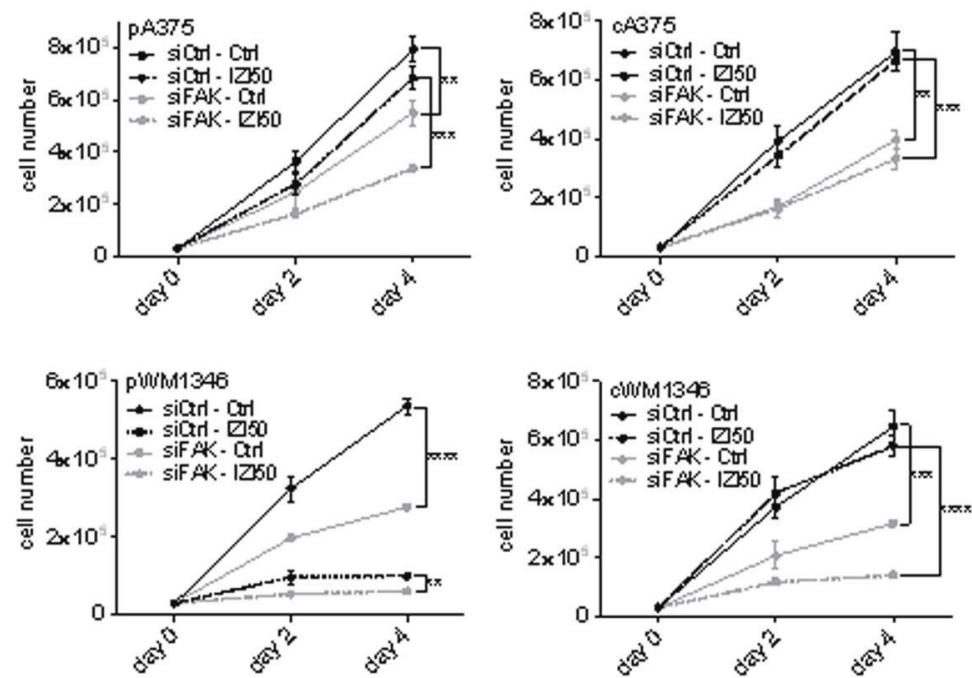

B
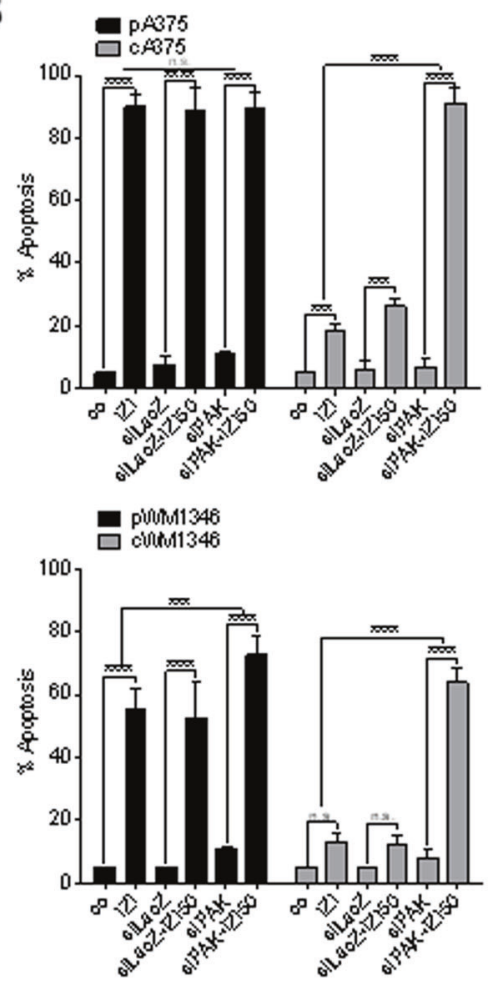

D
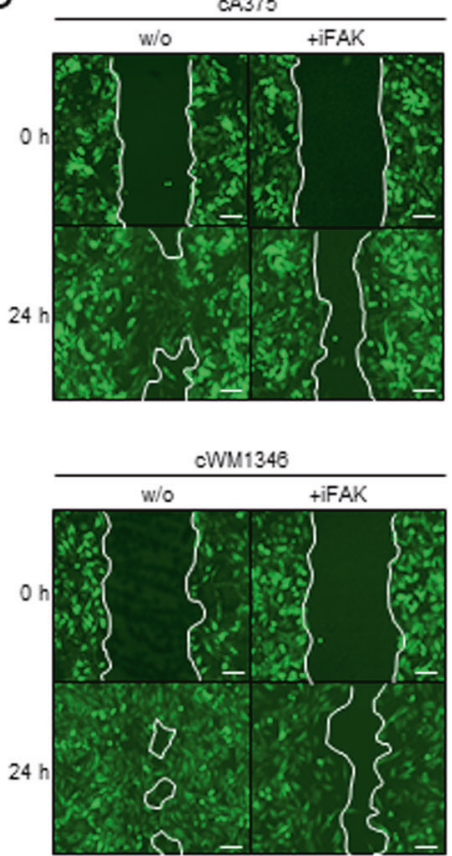

$E$
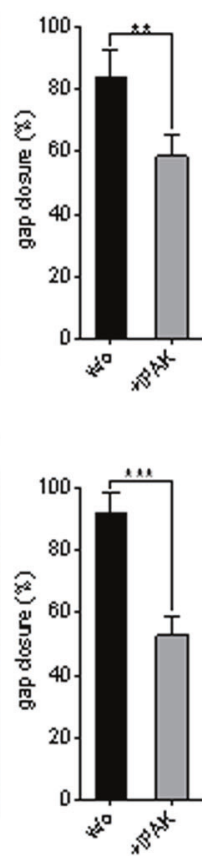

\section{D melanoma spheroids}

Melanoma spheroids were generated using the "hanging drop" method [24]. Briefly, (4-20) $\times 10^{4}$ GFP-expressing melanoma cells were resuspended in $5 \mathrm{ml}$ of RPMl containing $20-40 \%$ methocell, depending on cell line. 40 drops of $25 \mu$ l containing $200-1000$ cells were spotted on the lid of a $10 \mathrm{~cm}$ cell culture dish, inverted onto the dish filled with $10 \mathrm{ml}$ of $1 \times$ PBS, and incubated for 12 days at $37^{\circ} \mathrm{C}, 5 \% \mathrm{CO}_{2}$.

For analysis, mature spheroids were embedded into 3D dextran-based gel-matrices containing $6 \mathrm{nMol} / \mathrm{L}$ of thiol-reactive groups and thiol groups (3-D Life Dextran-CD Hydrogel SG, \#G93-1; Cellendes, Reutlingen, 
Fig. 7 TRAIL-resistance and tumor outgrowth of IZI5-conditioned MM cells depends on FAK. A FAK was depleted in parental ( $p$ ) and IZI5conditioned (c) A375 and WM1346 cells using RNA interference (siFAK), with siLacZ as scrambled control. Transient knockdown of FAK was monitored $24 \mathrm{~h}$ later by Western-blot analysis, with $\beta$-actin as loading control. One representative out of three independently performed experiments is shown. B Cells as in A were stimulated with IZI50 and apoptosis induction determined $24 \mathrm{~h}$ later using a CDDE $\left(n=3:{ }^{* * *} p \leq\right.$ $0.001 ;{ }^{* * * *} p \leq 0.0001 ;$ n.s. $=$ not significant). C Unstimulated cells as in A were subjected to a proliferation assay and cell numbers quantified at the indicated time points $\left(n=3:{ }^{* *} p \leq 0.01 ;{ }^{* * *} p \leq 0.001 ;{ }^{* * * *} p \leq 0.0001\right)$. D GFP-expressing IZI5-conditioned (c) A375 and WM1346 cells were left untreated or treated with the FAK inhibitor defactinib (iFAK, $5 \mu \mathrm{M})$. Migration of cells was monitored by scratch assay (scale bar $=100 \mu \mathrm{m})$ and gap closure quantified after $24 \mathrm{~h}\left(n=3:{ }^{* *} p \leq 0.01 ;{ }^{* *} p \leq 0.001\right)$. E GFP-expressing spheroids consisting of IZI5-conditioned (c) mutBRAF (A375, Malme3M) and mutNRAS (WM1346, WM1366) melanoma cells, respectively, were individually embedded into 3D dextran-based gelmatrices and stimulated with IZI50 and $5 \mu \mathrm{M}$ defactinib (iFAK) every other day over 8 days (scale bar $=200 \mu \mathrm{m}$ ). At day eight cell death was visualized by addition of $6.7 \mu \mathrm{g} / \mathrm{ml} \mathrm{PI}$. Confocal images of individual spheroids were taken at day one and eight and the tumor volume (green) versus tumor death (red) quantified at day eight $\left(n=3:{ }^{*} p \leq 0.05 ;{ }^{* *} p \leq 0.001\right)$.

Germany), according to the manufacturer's protocol. Dextran was used as the thiol-reactive polymer and crosslinked with a polyethylene glycol peptide conjugate (CD-Link), which contains a matrix-metalloprotease cleavable peptide that allows cells to cleave the crosslinker in order to spread and migrate throughout the gel. One single spheroid was injected per $30 \mu \mathrm{l} \mathrm{gel-matrix} \mathrm{in} \mathrm{a} \mathrm{volume} \mathrm{of} 5 \mu \mathrm{l}$ medium and incubated for $30 \mathrm{~min}$ at $37^{\circ} \mathrm{C}$. Subsequently, gels were covered with medium and drugs added every other day. Development of individual spheroids was monitored daily by confocal fluorescence microscopy (LSM 780/FCS inverse, Zeiss, Germany) equipped with a Plan-Apochromat $\times 10 / 0.45$ M27 objective, over 8 days. At day eight cell death of spheroids was visualized by the addition of $6.7 \mu \mathrm{g} / \mathrm{ml}$ propidium iodide (PI, Invitrogen) in PBS for $20 \mathrm{~min}$ at RT in the dark. After washing twice with PBS confocal images were taken. For the emitted green fluorescent, the laser emission peak was $488 \mathrm{~nm}$ (emission filter 499-597 nm). For the emitted red fluorescent, the laser emission peak was $561 \mathrm{~nm}$ (emission filter 606-686 nm).

\section{Proliferation and clonogenic outgrowth}

$(8-10) \times 10^{4}$ cells were seeded in 6-well plates and incubated with or without the indicated drug for $48 \mathrm{~h}$. Subsequently, cells were harvested, counted and re-seeded at the starting density every other day for three times. The progressive increase of cells was calculated as proliferation rate over time.

Clonogenic outgrowth was determined by seeding (1-6.5) $\times 10^{3}$ cells into 6-well plates for 8 days or until control cells had reached confluency. Subsequently, cells were stained with crystal violet $(0.1 \mathrm{w} / \mathrm{v}$ in $20 \%$ methanol) for $15 \mathrm{~min}$ at RT. After washing with PBS, crystal violet was dissolved from cells with $0.1 \mathrm{M} \mathrm{KH}_{2} \mathrm{PO}_{4} / \mathrm{EtOH}$ for $5 \mathrm{~min}$ at RT and color intensity of supernatants measured at $595 \mathrm{~nm}$ (Tecan M200, Tecan, Maennedorf, Switzerland).

\section{Scratch assay}

$6.5 \times 10^{5}$ GFP-expressing cells were plated onto cell culture dishes $(35 \times$ $10 \mathrm{~mm}$ ) at $90 \%$ confluency. After $24 \mathrm{~h}$, a $1-\mathrm{mm}$ wide scratch was inserted across the cell layer using a sterile $(10 \mu l)$ pipette tip. Closure of the scratch by cell migration was monitored over $48 \mathrm{~h}$ using smart fluorescent cell analyser (JuLIT ${ }^{\mathrm{TM}}$, VWR International GmbH, Germany).

\section{Determination of cell death}

Apoptosis was determined in a Cell Death Detection ELISA (CDDE, Roche, Mannheim, FRG). The enrichment of mono- and oligonucleosomes released into the cytosol is calculated: absorbance of samples/absorbance of control cells (Tecan M200). An enrichment factor of 2 corresponds to $10 \%$ apoptosis as determined by AnnexinV FACS analysis (FACSAria III, Becton Dickinson, Heidelberg, Germany).

Sensitivity towards kinase inhibitors was monitored by PI ( $1 \mu \mathrm{g} / \mathrm{ml})$ uptake using automated, image-based IncuCyte (Satorius, Goettingen, Germany) screening technology over a period of $48 \mathrm{~h}$, taking images every $2 \mathrm{~h}$.

\section{Plasmid and siRNA transfection}

For stable expression of GFP, $6.5 \times 10^{6}$ A375, Malme3M, WM1346 and WM1366 cells were electroporated with $25 \mu \mathrm{g}$ of pEGFP plasmid in $600 \mu \mathrm{l}$ medium containing $2 \%$ DMSO and maintained in selection medium containing $1 \mathrm{mg} /$ ml Geneticin G418 Sulfate (sc-29065; Santa Cruz Biotechnology Inc, Santa Cruz, CA, USA). GFP-positive cells were enriched by cell sorting (FACSAria III). For CRISPR/Cas9-mediated knock out of caspase-8 WM115 melanoma cells were transfected with pSpCas9(BB)-2a-GFP (PX458)-hCASP8 gRNA2.2 (clone \#1), and pSpCas9(BB)-2a-GFP (PX458)-hCASP8 gRNA3.1 (clone \#2), respectively, and
GFP-positive cells were enriched by cell sorting (FACS Aria III). Plasmids were kindly provided by Prof. Dr. Hamid Kashkar, Institute for Medical Microbiology, Immunology and Hygiene, University of Cologne, Germany.

Transient knockdown was facilitated by transfecting $5 \times 10^{4}$ cells with 40 pmol of the respective siRNA for FAK (\#6472, Cell Signaling, Danvers, MS, USA), and lacZ-5'-GCGGCUGCCGGAAUUUACCTT-3' (MWG Eurofins, Ebersberg, Germany) using Lipofectamine RNAiMax (Invitrogen) $24 \mathrm{~h}$ prior to stimulation.

\section{Western-blot analysis}

Cells were lysed in lysis buffer (50 mM HEPES, pH 7.5; $150 \mathrm{mM} \mathrm{NaCl} ; 10 \%$ glycerol; $1 \%$ Triton-X-100; $1.5 \mathrm{mM} \mathrm{MgCl}$; 1 mM EGTA; $100 \mathrm{mM} \mathrm{NaF} ; 10 \mathrm{mM}$ pyrophosphate, $0.01 \% \mathrm{NaN}_{3}$, phosSTOP ${ }^{\circ}$ and Complete ${ }^{\circ}$ ). For sub-cellular fractionation NE-PER ${ }^{\mathrm{TM}}$ Nuclear and Cytoplasmic Extraction Reagents (Thermo Scientific, Waltham, MS, USA) were used according to the manufacturer's instructions. After centrifugation, supernatants were collected and the protein content determined by DC Protein assay kit (BioRad, Hercules, CA, USA). $60-80 \mu \mathrm{g}$ of protein extracts were subjected to 4-15\% gradient SDS-PAGE (BioRad), blotted onto nitrocellulose membranes using the Trans-Blot ${ }^{\circ}$ Turbo $^{\mathrm{TM}}$ Transfer System (BioRad), blocked in $1 \%$ blocking solution (Roche) and incubated with antibodies directed against PARP, p53 (BD-Biosciences; \#551025, \#554293), caspase-8 (\#AG20B-0057; Adipogen, San Diego, CA, USA), caspase-3, caspase-9, AKT, p-AKT (S473), ERK1/2, p-ERK1/2(T202/Y204), FAK, p-FAK(Y397), cJun, p-cJun, PDK1, p-PDK1(S241), PI3K, p-PI3K(Y458-p85/Y199-p55), p38, p-p38(T180/412), pp53(S15), SRC, p-SRC(Y416), (Cell Signaling; \#9665, \#9502, \#2920, \#4060, \#9102, \#4376, \#3285, \#8556, \#9165, \#9261, \#3062, \#3061, \#4292, \#17366, \#9212, \#9216, \#9248, \#2108, \#6943), respectively, diluted in 0.5\% blocking solution. Equal loading was monitored by re-probing membranes with antibodies against $\beta$-actin, GADPH (Cell Signaling: \#4970, \#2118), or atubulin (Thermo Scientific: \#MS-581-P1). HRP-conjugated secondary antibodies were purchased from GE-Healthcare (Anti-mouse-HRP, \#NA931; Anti-rabbit-HRP, \#NA934). Bands were visualized by applying chemiluminescence SuperSignal ${ }^{\circ}$ detection systems (Thermo Scientific).

\section{Flow cytometry analysis}

$5 \times 10^{5}$ cells were blocked in PBS/2\% BSA for $30 \mathrm{~min}$ and incubated with a fluorescently labeled anti aV $\beta 3$ integrin antibody (Alexa Fluor 488 antihuman CD51/61, \#304408, Biolegend, San Diego, CA, USA) at $1 \mathrm{mg} / \mathrm{ml}$ in $\mathrm{PBS} / 2 \% \mathrm{BSA}$, for $1 \mathrm{~h}$ on ice. After washing cells were subjected to FACS analysis (FACSAria III). Excitation wavelength used was $488 \mathrm{~nm}$. The emitted green fluorescence (Imax $520 \mathrm{~nm}$ ) was detected using (FL1) band-pass filter.

\section{RNA extraction and RNAseq analysis}

RNA was extracted from $5 \times 10^{6}$ cells using RNeasy Mini-Kit (Qiagen, Minden, Germany) according to the manufacturer's protocol.

Purity of RNA was assessed by determining the ratio of absorbance at $260 \mathrm{~nm}$ and $280 \mathrm{~nm}$ (M200 Tecan). RNA integrity was investigated with Agilent 2100 Bioanalyzer (Agilent Technologies, Palo Alto, CA, USA). RNA sequencing was performed according to the previously published protocols [48]. Briefly, $1 \mu \mathrm{g}$ of total RNA was used for the library preparation using TruSeq stranded mRNA library preparation kit (Illumina, San Diego, CA, USA). The libraries were quantified using Qubit dsDNA HS assay kit (ThermoFisher Scientific, Waltham, MA USA) and size distribution was determined using Agilent 2100 Bioanalyzer. All prepared samples were pooled in equimolar concentration and sequenced at LCSB sequencing platform using NextSeq500 (Illumina, San Diego, CA, USA). Reads handling from trimming to feature counting was performed using the workflow manager Snakemake (v5.20.1 [49]) and our RNA-seq template (release v0.2 
A
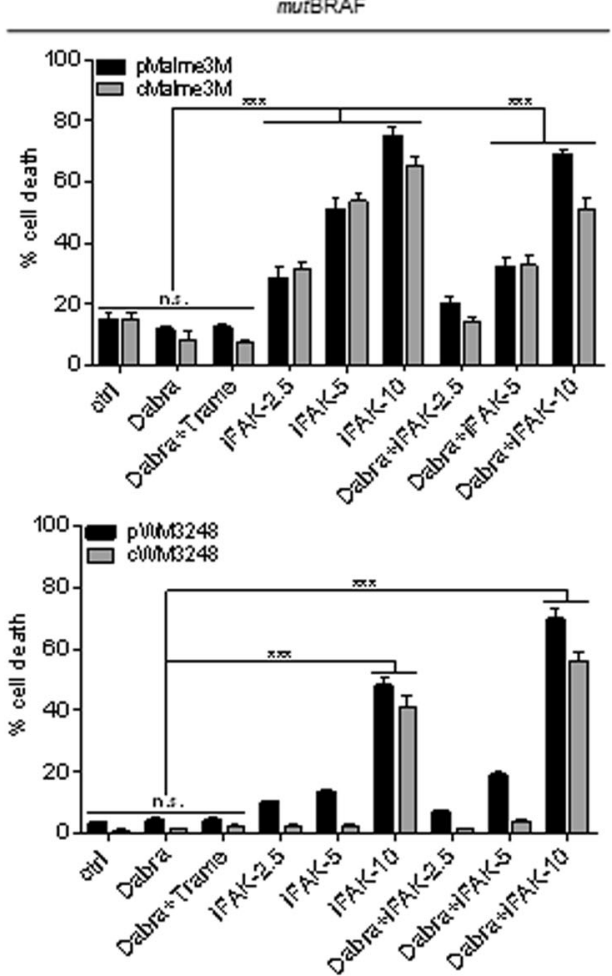

B

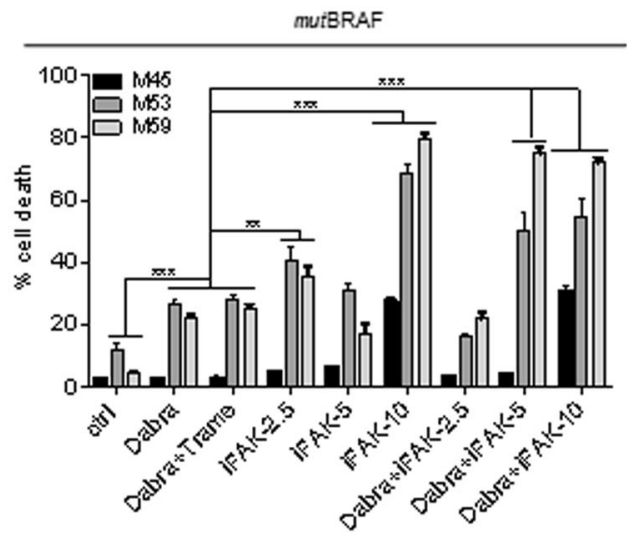

C

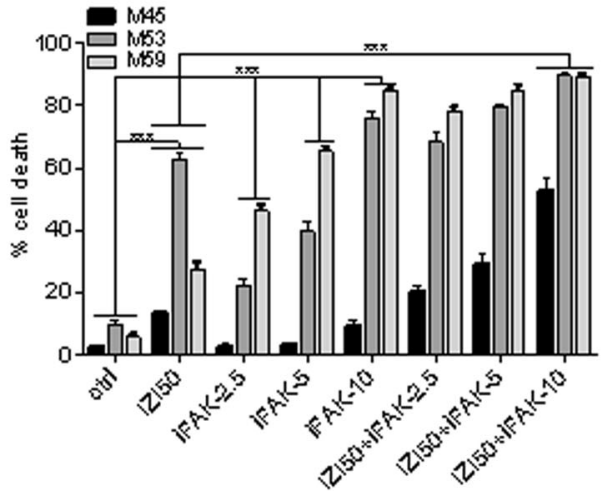

freely available at https://git-r3lab.uni.lu/aurelien.ginolhac/snakemake-rnaseq). Briefly, the trimming for adapter sequences was done using AdapterRemoval (v2.3.1 [50]) using the following settings: minimal length to retained reads set to $35 \mathrm{bp}$ and adapter 1 set to AGATCGGAAGAGCACACGTCTGAACTCCAGTCAC. Reads were aligned to the human reference genome GRCh38 with the ensembl gene annotation version 100 using
mUINRAS
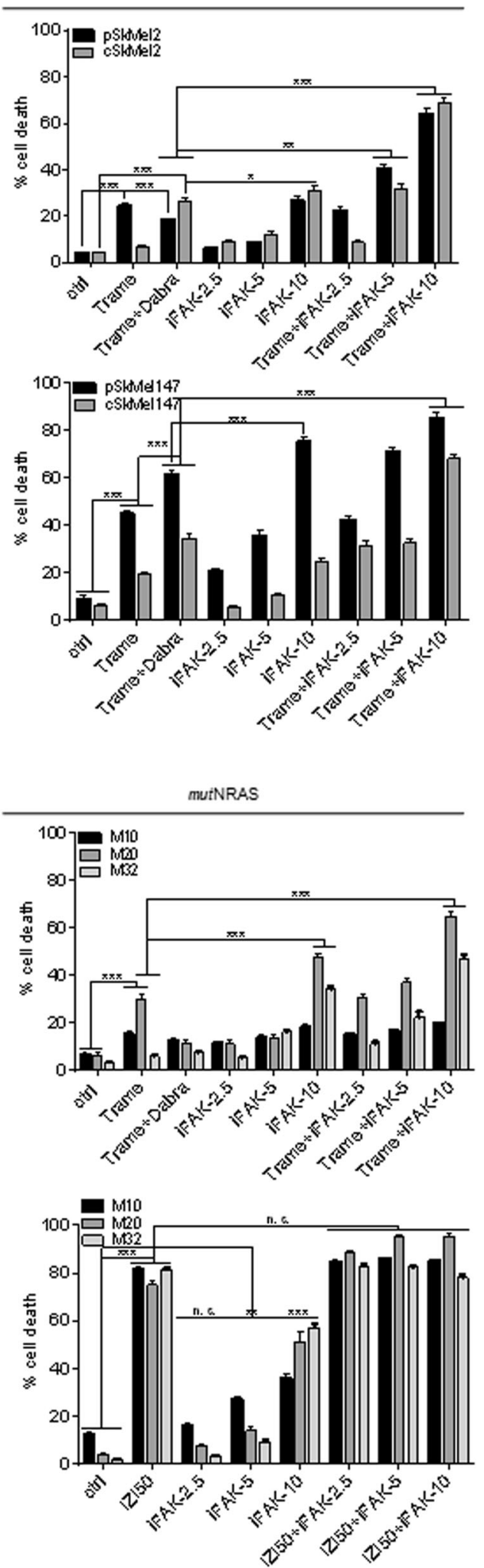

STAR (v2.7.4a [51]). Read count generation was performed using featureCounts from RSubread (v2.2.2 [52]) ignoring multimapping reads. Differential expression analysis was performed using raw counts and the DESeq2 package (v1.28.1 [25]. Log $_{2}$-fold change ( $\log _{2} \mathrm{FC}$ ) shrinkage was performed using apeglm to preserve large effects of true positives ( $\mathrm{v} 1.10 .0$ [53]). Plots were created using ggplot2 (v3.3.2 Wickham 2016), ggforce 
Fig. 8 FAK inhibition sensitizes resistant mutBRAF and mutNRAS melanoma to mutation-specific targeted kinase inhibition. A Parental mutBRAF Malme3M and WM3248 MM cells were conditioned to $1 \mu \mathrm{M}$ BRAF inhibitor dabrafenib (Dabra); mutNRAS SkMel2 and SkMel147 cells to $1 \mathrm{nM}$ MEK inhibitor trametinib (Trame). mutBRAF parental ( $p$ ) and conditioned (c) cells were treated with $10 \mu \mathrm{M}$ dabrafenib, dabrafenib + $1 \mu \mathrm{M}$ trametinib, 2.5, 5, or $10 \mu \mathrm{M}$ of the iFAK defactinib alone and in combination with $10 \mu \mathrm{M}$ dabrafenib. Parental (p) and conditioned (c) mutNRAS cells were treated with $1 \mu \mathrm{M}$ trametinib, trametinib $+10 \mu \mathrm{M}$ dabrafenib, $2.5,5$ or $10 \mu \mathrm{M}$ of the iFAK defactinib alone and in combination with trametinib. Cell death was monitored by PI $(1 \mu \mathrm{g} / \mathrm{ml})$ uptake using IncuCyte live-cell analysis for $48 \mathrm{~h}$. B Three mutBRAF (M45, M53, M59) and three mutNRAS (M10, M20, M32) cell samples freshly isolated from patients with relapsed melanoma metastases were treated as in A according to their mutation status. Cell death was monitored by PI (1 $\mu \mathrm{g} / \mathrm{ml})$ uptake using IncuCyte technology for $48 \mathrm{~h}$. C The same patient-derived metastatic cell samples as in (B) were treated with IZI50, 2.5, 5, or $10 \mu \mathrm{M}$ of the iFAK defactinib alone and in combination with IZI50. Cell death was monitored by PI $(1 \mu \mathrm{g} / \mathrm{ml})$ uptake using IncuCyte technology for 48 h. For each experiment $n=3$ is shown $\left({ }^{*} p \leq\right.$ $0.05 ;{ }^{* *} p \leq 0.01 ;{ }^{* *} p \leq 0.001 ;$ n.s. $=$ not significant).

(v0.3.3 Pedersen 2020, https://cran.r-project.org/web/packages/ggforce/ index.html) and ComplexHeatmap (v2.7.8.1 [54]). All config files, code and packages references are available at the following address: https:// github.com/sysbiolux/TRAIL_IZI_Melanoma. RNAseq data are available in EBI's ArrayExpress under accession number E-MTAB-10669 (reviewer link: http://www.ebi.ac.uk/arrayexpress/help/how_to_search_private_data.html; username: Reviewer_E-MTAB-10669; password: OrqyKI1b).

\section{Quantification and statistical analysis}

Unless stated otherwise, results of Cell Death Detection ELISA and flow cytometry analysis are presented as mean \pm SD of three independently performed experiments. Western-blot analysis represents one out of three independently performed experiments. Statistical analysis was performed with unpaired Student's $t$ test using GraphPad PRISM 6 software (https:// www.graphpad.com).

Quantification of 3D melanoma spheroids (tumor mass) was performed calculating the area of the green fluorescent spheroid and the red PI stained cells. For the migration and invasion analysis, the total area (in pixels) of the cells was measured, whereas for the scratch assay the size of the gap was determined.

All the quantification measurements of spheroids, migration, scratch, and invasion assays were performed by using Fiji software (https://fiji.sc).

\section{DATA AVAILABILITY}

RNAseq data are available in EBl's ArrayExpress under accession number E-MTAB-10669 (reviewer link: http://www.ebi.ac.uk/arrayexpress/help/how_to_search_private_data.html; username: Reviewer_E-MTAB-10669; password: OrqyKI1b).

\section{REFERENCES}

1. Niessner H, Schmitz J, Tabatabai G, Schmid AM, Calaminus C, Sinnberg T, et al. PI3K Pathway inhibition achieves potent antitumor activity in melanoma brain metastases in vitro and in vivo. Clin Cancer Res. 2016;22:5818-28.

2. Niessner $H$, Sinnberg T, Kosnopfel C, Smalley KSM, Beck D, Praetorius $C$, et al. BRAF inhibitors amplify the proapoptotic activity of MEK inhibitors by inducing ER stress in NRAS-mutant melanoma. Clin Cancer Res. 2017;23:6203-14.

3. Paluncic J, Kovacevic Z, Jansson PJ, Kalinowski D, Merlot AM, Huang ML, et al. Roads to melanoma: Key pathways and emerging players in melanoma progression and oncogenic signaling. Biochim Biophys Acta. 2016;1863:770-84.

4. Long GV, Flaherty KT, Stroyakovskiy D, Gogas H, Levchenko E, de BF, et al. Dabrafenib plus trametinib versus dabrafenib monotherapy in patients with metastatic BRAF V600E/K-mutant melanoma: long-term survival and safety analysis of a phase 3 study. Ann Oncol. 2019;30:1848.

5. Gotwals P, Cameron S, Cipolletta D, Cremasco V, Crystal A, Hewes B, et al. Prospects for combining targeted and conventional cancer therapy with immunotherapy. Nat Rev Cancer. 2017;17:286-301.

6. Luke JJ, Flaherty KT, Ribas A, Long GV. Targeted agents and immunotherapies: optimizing outcomes in melanoma. Nat Rev Clin Oncol. 2017;14:463-82.

7. Dummer R, Lebbe C, Atkinson V, Mandala M, Nathan PD, Arance A. et al. Combined PD-1, BRAF and MEK inhibition in advanced BRAF-mutant melanoma: safety run-in and biomarker cohorts of COMBI-i. Nat Med. 2020;26:1557-63.

8. Davis EJ, Perez MC, Ayoubi N, Zhao S, Ye F, Wang DY, et al. Clinical correlates of response to anti-PD-1-based therapy in patients with metastatic melanoma. J Immunother. 2019;42:221-7.

9. Ju RJ, Stehbens SJ, Haass NK. The role of melanoma cell-stroma interaction in cell motility, invasion, and metastasis. Front Med. 2018;5:307.

10. Humphries JD, Byron A, Humphries MJ. Integrin ligands at a glance. J Cell Sci. 2006;119:3901-3.
11. Hood JD, Cheresh DA. Role of integrins in cell invasion and migration. Nat Rev Cancer. 2002;2:91-100

12. Carragher NO, Frame MC. Focal adhesion and actin dynamics: a place where kinases and proteases meet to promote invasion. Trends Cell Biol. 2004;14:241-9.

13. Cooper J, Giancotti FG. Integrin signaling in cancer: mechanotransduction, stemness, epithelial plasticity, and therapeutic resistance. Cancer Cell. 2019;35:347-67.

14. Ramsay AG, Marshall JF, Hart IR. Integrin trafficking and its role in cancer metastasis. Cancer Metastasis Rev. 2007;26:567-78.

15. Yoon H, Dehart JP, Murphy JM, Lim ST. Understanding the roles of FAK in cancer: inhibitors, genetic models, and new insights. J Histochem Cytochem. 2015;63:114-28.

16. Tai YL, Chen LC, Shen TL. Emerging roles of focal adhesion kinase in cancer. Biomed Res Int. 2015;2015:690690.

17. Zhao X, Guan JL. Focal adhesion kinase and its signaling pathways in cell migration and angiogenesis. Adv Drug Deliv Rev. 2011;63:610-5.

18. Golubovskaya VM, Cance WG. FAK and p53 protein interactions. Anticancer Agents Med Chem. 2011;11:617-9.

19. Lim ST, Chen $X L$, Lim Y, Hanson DA, Vo TT, Howerton $K$, et al. Nuclear FAK promotes cell proliferation and survival through FERM-enhanced p53 degradation. Mol Cell. 2008;29:9-22.

20. Hutt M, Marquardt L, Seifert O, Siegemund M, Muller I, Kulms D, et al. Superior properties of Fc-comprising scTRAIL fusion proteins. Mol Cancer Ther. 2017;16:2792-802.

21. Siegemund $M$, Schneider $F$, Hutt $M$, Seifert $O$, Muller I, Kulms D, et al. IgG-singlechain TRAIL fusion proteins for tumour therapy. Sci Rep. 2018;8:7808.

22. Del MG, Lucarelli $P$, Muller I, De LS, Zinoveva A, Hutt M, et al. Systemic network analysis identifies XIAP and IkappaBalpha as potential drug targets in TRAIL resistant BRAF mutated melanoma. NPJ Syst Biol Appl. 2018;4:39.

23. Henry CM, Martin SJ. Caspase-8 acts in a non-enzymatic role as a scaffold for assembly of a pro-inflammatory "FADDosome" Complex upon TRAIL stimulation. Mol Cell. 2017;65:715-29.

24. Vorsmann H, Groeber F, Walles H, Busch S, Beissert S, Walczak H, et al. Development of a human three-dimensional organotypic skin-melanoma spheroid model for in vitro drug testing. Cell Death Dis. 2013;4:e719.

25. Love MI, Huber W, Anders S. Moderated estimation of fold change and dispersion for RNA-seq data with DESeq2. Genome Biol. 2014;15:550.

26. letswaart R, Gyori BM, Bachman JA, Sorger PK, Churchman LS. GeneWalk identifies relevant gene functions for a biological context using network representation learning. Genome Biol. 2021;22:55.

27. Sonoda $Y$, Matsumoto $Y$, Funakoshi M, Yamamoto D, Hanks SK, Kasahara T. Antiapoptotic role of focal adhesion kinase (FAK). Induction of inhibitor-of-apoptosis proteins and apoptosis suppression by the overexpression of FAK in a human leukemic cell line, HL-60. J Biol Chem. 2000;275:16309-15.

28. Li K, van Delft MF, Dewson G. Too much death can kill you: inhibiting intrinsic apoptosis to treat disease. EMBO J. 2021;40:e107341.

29. Lu H, Liu S, Zhang G, Bin W, Zhu Y, Frederick DT, et al. PAK signalling drives acquired drug resistance to MAPK inhibitors in BRAF-mutant melanomas. Nature 2017;550:133-6.

30. Miller MA, Oudin MJ, Sullivan RJ, Wang SJ, Meyer AS, Im H, et al. Reduced proteolytic shedding of receptor tyrosine kinases is a post-translational mechanism of kinase inhibitor resistance. Cancer Discov. 2016;6:382-99.

31. Shen $\mathrm{CH}$, Kim SH, Trousil S, Frederick DT, Piris A, Yuan P, et al. Loss of cohesin complex components STAG2 or STAG3 confers resistance to BRAF inhibition in melanoma. Nat Med. 2016;22:1056-61.

32. Wattson DA, Sullivan RJ, Niemierko A, Merritt RM, Lawrence DP, Oh KS, et al. Survival patterns following brain metastases for patients with melanoma in the MAP-kinase inhibitor era. J Neurooncol. 2015;123:75-84.

33. Dimberg LY, Anderson CK, Camidge R, Behbakht K, Thorburn A, Ford HL. On the TRAIL to successful cancer therapy? Predicting and counteracting resistance against TRAIL-based therapeutics. Oncogene 2013;32:1341-50. 
34. Czarnecka AM, Bartnik E, Fiedorowicz M, Rutkowski P. Targeted therapy in melanoma and mechanisms of resistance. Int J Mol Sci. 2020;21:4576.

35. Rambow F, Marine JC, Goding CR. Melanoma plasticity and phenotypic diversity: therapeutic barriers and opportunities. Genes Dev. 2019;33:1295-318.

36. Golubovskaya VM, Kweh FA, Cance WG. Focal adhesion kinase and cancer. Histol Histopathol. 2009;24:503-10.

37. Rigiracciolo DC, Cirillo F, Talia M, Muglia L, Gutkind JS, Maggiolini M, et al. Focal adhesion kinase fine tunes multifaced signals toward breast cancer progression. Cancers. 2021;13:645.

38. Kong D, Chen F, Sima NI. Inhibition of focal adhesion kinase induces apoptosis in bladder cancer cells via Src and the phosphatidylinositol 3-kinase/Akt pathway. Exp Ther Med. 2015;10:1725-31.

39. Schober M, Fuchs E. Tumor-initiating stem cells of squamous cell carcinomas and their control by TGF-beta and integrin/focal adhesion kinase (FAK) signaling. Proc Natl Acad Sci USA. 2011;108:10544-49.

40. Grossmann J, Artinger M, Grasso AW, Kung HJ, Scholmerich J, Fiocchi C, et al. Hierarchical cleavage of focal adhesion kinase by caspases alters signal transduction during apoptosis of intestinal epithelial cells. Gastroenterology 2001;120:79-88

41. Sasaki H, Kotsuji F, Tsang BK. Caspase 3-mediated focal adhesion kinase processing in human ovarian cancer cells: possible regulation by $\mathrm{X}$-linked inhibitor of apoptosis protein. Gynecol Oncol. 2002;85:339-50.

42. Wick W, Wick A, Schulz JB, Dichgans J, Rodemann HP, Weller M. Prevention of irradiation-induced glioma cell invasion by temozolomide involves caspase 3 activity and cleavage of focal adhesion kinase. Cancer Res. 2002;62:1915-9.

43. Wen LP, Fahrni JA, Troie S, Guan JL, Orth K, Rosen GD. Cleavage of focal adhesion kinase by caspases during apoptosis. J Biol Chem. 1997;272:26056-61.

44. Kurenova E, Xu LH, Yang X, Baldwin AS Jr., Craven RJ, Hanks SK, et al. Focal adhesion kinase suppresses apoptosis by binding to the death domain of receptor-interacting protein. Mol Cell Biol. 2004;24:4361-71.

45. van de Water B, Nagelkerke JF, Stevens JL. Dephosphorylation of focal adhesion kinase (FAK) and loss of focal contacts precede caspase-mediated cleavage of FAK during apoptosis in renal epithelial cells. J Biol Chem. 1999;274:13328-37.

46. Muller I, Strozyk E, Schindler S, Beissert S, Oo HZ, Sauter T, et al. Cancer cells employ nuclear Caspase-8 to overcome the p53-dependent G2/M checkpoint through cleavage of USP28. Mol Cell. 2020;77:970-84.

47. Webster MR, Fane ME, Alicea GM, Basu S, Kossenkov AV, Marino GE, et al. Paradoxical role for wild-type $\mathrm{p} 53$ in driving therapy resistance in melanoma. Mol Cell. 2020;77:633-44.

48. Gerard D, Schmidt F, Ginolhac A, Schmitz M, Halder R, Ebert P, et al. Temporal enhancer profiling of parallel lineages identifies AHR and GLIS1 as regulators of mesenchymal multipotency. Nucleic Acids Res. 2019;47:1141-63.

49. Molder $F$, Jablonski KP, Letcher $B$, Hall MB, Tomkins-Tinch $\mathrm{CH}$, Sochat $\mathrm{V}$, et al. Sustainable data analysis with Snakemake. F1000Res. 2021;10:33.

50. Schubert M, Lindgreen S, Orlando L. AdapterRemoval v2: rapid adapter trimming, identification, and read merging. BMC Res Notes. 2016;9:88.

51. Dobin A, Davis CA, Schlesinger F, Drenkow J, Zaleski C, Jha S, et al. STAR: ultrafast universal RNA-seq aligner. Bioinformatics 2013;29:15-21.

52. Liao Y, Smyth GK, Shi W. The R package Rsubread is easier, faster, cheaper and better for alignment and quantification of RNA sequencing reads. Nucleic Acids Res. 2019;47:e47.

53. Zhu A, Ibrahim JG, Love MI. Heavy-tailed prior distributions for sequence count data: removing the noise and preserving large differences. Bioinformatics 2019;35:2084-92.

54. Gu Z, Eils R, Schlesner M. Complex heatmaps reveal patterns and correlations in multidimensional genomic data. Bioinformatics 2016;32:2847-9.

\section{ACKNOWLEDGEMENTS}

RNAseq and transcriptomic analysis were carried out using the HPC facilities of the University of Luxembourg (Varrette et al. 2014, https://hpc.uni.lu). This work was funded by the Federal Ministry of Education and Research (FKZ 031A423A).

\section{AUTHOR CONTRIBUTIONS}

G.D.M., S.R., and S.S. conducted all cell-based experiments. L.S. and R.H. performed RNAseq analysis, A.G. and L.P. managed database and GeneWalk analysis. R.E.K generated and provided IZI1551. D.K. and T.S. conceived the project and supervised research. S.B. and all authors contributed to experimental design and manuscript writing

\section{FUNDING}

Open Access funding enabled and organized by Projekt DEAL.

\section{COMPETING INTERESTS}

The authors declare no competing interests.

\section{ETHICS STATEMENT}

The usage of patient material (metastasis) for biochemical analysis was approved by the ethics committee of the TU-Dresden (EK 335082018) and informed consent was obtained from all patients.

\section{ADDITIONAL INFORMATION}

Supplementary information The online version contains supplementary material available at https://doi.org/10.1038/s41419-022-04502-8.

Correspondence and requests for materials should be addressed to Dagmar Kulms.

Reprints and permission information is available at http://www.nature.com/ reprints

Publisher's note Springer Nature remains neutral with regard to jurisdictional claims in published maps and institutional affiliations.

Attribution 40 International license, which permits use, sharing, adaptation, distribution and reproduction in any medium or format, as long as you give appropriate credit to the original author(s) and the source, provide a link to the Creative Commons license, and indicate if changes were made. The images or other third party material in this article are included in the article's Creative Commons license, unless indicated otherwise in a credit line to the material. If material is not included in the article's Creative Commons license and your intended use is not permitted by statutory regulation or exceeds the permitted use, you will need to obtain permission directly from the copyright holder. To view a copy of this license, visit http://creativecommons. org/licenses/by/4.0/.

(c) The Author(s) 2022 